\title{
Über die Umwandlung der Maleïnsäure in Fumarsäure
} von

\author{
Zd. H. Skraup.
}

Aus dem chemischen Institute der k. k. Universität in Graz.

(Vorgelegt in der Sitzung am 16. April 1891.)

In neuester 'Zeit hat die Umlagerung der Maleïnsärtre in die isomere Fumarsäure bei der Discussion stereochemischer Fragen eine nicht unwichtige Rolle gespielt.

J. Wislicenus fasst sie als einen Specialfall jener grossen Zahl von Umlagerungen auf, die nach seiner geistreichen Hypothese derart verlaufen, dass intermediäre Additionsproducte entstehen, die eine stereochemische Drehung erfahren, dann die friiher aufgenommenen Elemente wieder abspalten und dadurch Isomere der ursprïnglichen Verbindungen liefern, mit welch' letzteren jene structurchemisch nicht, aber stereochemisch identisch sind. Malë̈nsäure und Salzsäure sollen z. B. Chlorbernsteinsäure geben, die nach der Drehung Salzsäure abspaltet und Fumarsäure liefert.

Gegen diese Annahme haben Ansehiitz und Fittig den Einwand erboben, dass in mehreren Fällen die von Wislicenus angenommenen intermediären Additionsproducte unter solchen Bedingungen beständig sind, unter welchen sie gemäss der Hypothese von Wislicenus entstehen und wieder zerfallen sollten; Anschuitz hat dies für die Chlorbernsteinsäure, Fittig für die Dibrombernsteinsäure hervorgehoben, welche nach der Hypothese von Wislicenus bei den Umwandlungen durch Salzsäure und Brom die Zwischenglieder sein sollen.

Da, wie schon erwähnt, die im Titel genannte Reaction ein Einzelnfall aus einer grossen Zahl chemischer Processe ist und 
die für ein Beispiel festgestellten Bedingungen allgemeiner Natur sein könnten, habe ich eine Untersuchung der so viel discutirten Umlagerung in der Weise unternommen, dass schon bekannte Reactionen und andere, die nen aufgestossen sind, nicht nur qualitativ, sondern anch quantitativ verfolgt wurden.

Die wichtigsten Ergebnisse der Versuche seien dem experimentellen Theil vorangeschickt.

Die Umwandlung der Maleïnsäure in Fumarsäure gelingt unter dem Einfluss von Säuren, von Wasser, sie ist durch nebenher laufende chemische Processe möglich.

Die Umwandlungsfähigkeit dürfte den meisten Säuren zukommen.

Bei sehr verdünnten Säuren (balbnormalen) ist jene möglicherweise eine sogenannte Säurewirkung, ähnlich wie bei der Verseifung von Estern; sie erfolgt aber so langsam, dass ein ganz sicherer Nachweis nicht möglich war.

Concentrirte Säuren (doppeltnormale und stärkere) wirken atisserordentlich ungleich, manche der sogenannten stärkeren schon bei gewöhnlicher Temperatur, andere und die schwächeren erst bei erhöhter, die starken und schwachen untereinander aber so ungleich rasch, dass eine Beziehung zwischen dem Leitungsvermögen und der Umwandlung bestimmt ausgeschlossen ist, wie iberdies speciell angestellte Versuche gezeigt haben.

Anch solche Säuren, von welchen im Sinne der Theorie von Wislicenus Additionsproducte kaum annehmbar sind, wie Schwefelsäure, Salpetersäure, Oxalsäure, bewirken die Umwandlung.

Maleïnsäure mit Wasser unter Druck erhitzt, wird in Fumarsäure verwandelt, Äpfelsäure, die als intermediäres Product in Betracht kommen könnte, wird unter analogen Verhältnissen in Fumarsäure nicht übergefuhrt, ebenso nicht, wenn man sie mit Säuren derselben Concentration behandelt, die Maleïnsäure leicht umlagert. Es ist desshalb nicht anzunehmen, dass die Äpfelsäure bei der Verwandlung durch Säuren oder mit Wasser allein als Zwischenglied auftritt. Die Wislicenus'sche Vorstellung ist, wenigstens in ihrer jetzigen Form, gewiss nicht richtig, man wollte denn annehmen, dass die von ihr gemeinten Additionsproducte im Entstehungszustande sich ganz anders verhalten als 
sonst, eine Annahme, die schon desshalb zu vermeiden ist, weil man mit ihr Alles beweisen könnte.

Und doch spielen bei der Umwandlung der Maleïnsäure Additionsproducte eine wichtige Rolle. Es ist von Fittig für die Einwirkung von Bromwasserstoffsäure, von Wislicenus für die Einwirkung von Brom schon nachgewiesen worden, dass neben Fumarsäure die additionellen Verbindungen der Maleïnsäure entstehen. Dasselbe gilt nun anch für die Einwirkung von Salzsäure, von Jodwasserstoffsäure und von Wasser, und eine möglichst genaue Verfolgung der Reactionen hat gezeigt, dass die beiden Vorgänge, die Umlagerung der Maleïnsäure und der Übergang. in die gesättigte Verbindung, stets parallel laufen. Demnach vermittelt der letztere katalytisch die Umagerung.

Solche katalytische Wirkung ist auch bei Processen zu beobachten, bei welchen die Maleïnsäure wohl umgelagert, aber sonst chemisch nicht verändert wird.

So bei der Zerlegung maleïnsaurer Salze durch Schwefelwasserstoff. Alle bisher untersuchten Salze der Schwermetalle scheiden, mit Schwefelwasserstoff behandelt, wohl den grössten Theil der Maleïnsäure unverändert ab, einen anderen aber als Fumarsäure, und der Procentgehalt letzterer steht mit der Natur des Metalls in directer Beziehung. Im experimentellen Theil wird der Nachweis, dass secundäre Reactionen dieses Resultat nicbt beeinflussen, im Detail erbracht werden. Dass auch hier von einem Vorgang im Sinne der Hypothese von Wislicenus nicht die Rede sein kann, geht daraus hervor, dass Schwefelwasserstoff auf Maleïnsäure so gut wie ohne Wirkung ist, ebenso wie Kohlendioxyd und Schwefligsäureanhydrid. Wirken aber Schwefelwasserstoff und Schwefeldioxyd auf Maleïnsäure ein, so wird viel Fumarsäure gebildet. Besondere Versuche haben festgestellt, dass anch die labilsten additionellen Verbindungen von Maleïnsätre mit einem der beiden Gase keinerlei Einfluss haben könnten. Die verschiedenen Processe, welchen die Umwandlung der Maleïnsäure zuzuschreiben ist und denen ein katalytischer Einfluss zukommt, sind sehr mannigfaltig, sie haben bloss das gemein, dass sie Änderungen der chemischen Energie herbeiführen und soweit ein Urtheil möglich ist, exothermisch verlaufen. Der Übergang der Maleïnsäure in Fumarsäure ist aber anch exothermisch, und 
demzufolge sind ähnliche Vorstellungen, wie über die Darstellung: von Jodwasserstoffsäure aus Jod und Schwefelwasserstoff, hier unannehmbar.

Auffallend ist weiter, dass nicht alle exothermischen Processe die gleiche Wirkung üben, denn die Zerlegung von Essigsüureanbydrid und verschiedenen Estern durch Wasser hat keinerlei Wirkung auf Maleïnsäure. Man muss demnach sonst ähnliche chemische Processe in solche unterscheiden, welche eine Energieänderung in der Maleïnsäure, d. i. Umlagerung in Fumarsäure hervorzurufen vermögen, und in solche, welchen eine derartige Wirkung abgeht.

Diese Thatsachen ausreichend za erklären, wird sehr schwierig sein.

An diesem Orte möchte ich bloss daranf hinweisen, dass die erwähuten Verhältnisse eine gewisse Ähnlichkeit mit den Erscheinungen der Resonanz oder auch denen der Influenz besitzen, und in rohen Umrissen trifft vielleicht das Bild zu: bei manchen chemischen Processen entstehen Schwingungen, die im Stande sind, in anderen Molekülen, welche im gewöhnlichen Sinne chemisch unbetheiligt sind, wieder Schwingungen zu erzengen, welche dann, sei es fül sich, sei es unterstittzt dureh andere Momente, wie Wärmeschwingungen, eine totale Änderung in der Structur herbeifuihren.

\section{Bestimmnng der Fumarsäure.}

Die Mehrzahl der untersuchten Reactionen ist quantitativ verfolgt, $d$. h. bei ihnen festgestellt worden, wieviel Maleïnsäure in Fumarsäure uibergegangen ist. Da die Fumarsäure viel weniger löslich ist als die Maleïnsäure, hat es keinerlei Schwierigkeit, Messungen vorzunehmen, wenn alle Lösungen, die bei einer Reaction zusammenwirken, zuvor mit Fumarsäure gesättigt waren. Wo nicht ausdrücklich das Gegentheil angeführt ist, wurden solche gesättigte Lösungen verwendet. Die ausgefallene Fumarsäure ist, wo nicht anders angegeben, filtrirt, mit gesättigter wässeriger Fumarsäurelösung, für die in der Folge der Name Fumarwasser in Anwendung kommen soll, gewaschen, dann in heissem Wasser gelöst und durch Titration mit ZehntelnormalNatronlange und Phenolpbtaleïn bestimmt worden. 
Fast in allen untersuchten Fällen habe ich die Fumarsäure durch qualitative Reactionen identificirt, ibre Zusammensetzung einigenal durch Titration gewogener Substanz, mitunter auch durch die Elementaranalyse controlirt. Bei der Einwirkung von Wasser auf Maleïnsäure, wo den Titre secundäre Reactionen nicht beeinflussen, konnte die gebildete und auskrystallisirte Fumarsäure durch Vergleichung des anfänglichen und des schliesslichen Titres gefunden werden.

Die mitgetheilten Zahlen beanspruchen keine scharfe Geltung, die Methode hat viele Fehlerquellen, insbesondere die, dass die Fumarsäure nur langsam auskrystallisirt: Immerhin lassen sich dort, wo die Abweichungen zweifellos viel grösser sind als die Versuchsfehler; Schlïsse ziehen; dass bei Einhaltung gleicher Bedingungen die Resultate nicht schlecht übereinstimmen und desshalb einigen Verlass bieten, zeigen u. A. die Mengen von Fumarsäure, die sich beim Erhitzen von je $5 \mathrm{~cm}^{3} 5 \%$ iger Maleïnsäurelösung auf $135^{\circ}$ durch drei Stunden abschieden, bei welchen ganz verschiedene Maleïnsäureproben des Handels in Anwendung kamen, und bei welchen das Auskrystallisiren in einigen Fällen die fünffache Zeit währte als in anderen.

Die in sechs Fällen abgeschiedene Fumarsäure verbranchte $8 \cdot 7,8 \cdot 1,7 \cdot 0,6 \cdot 2,7 \cdot 0,9 \cdot 5 \mathrm{~cm}^{3} 1 / 10 \mathrm{NaOH}$.

\section{Einwirknng von Wasser auf Maleïnsäure.}

Bei gewöbnlichem Druck and Temperaturen bis $100^{\circ}$ wird Maleïnsäure durch Wasser nicht, jedenfalls nicht in dem Masse in Fumarsäure ïbergeführt, dass leiztere qualitativ oder quantitativ nachzuweisen wäre.

Bei höherer Temperatur unter verstärktem Druck geht aber die Umwandlung vor sich.

Vier Röhren mit je $1 \mathrm{~cm}^{3} 25 \%$ Maleïnsäurelösung nnd $4 \mathrm{~cm}^{3}$ Fumarwasser wurden 12, 9, 6 und 3 Stunden auf $135^{\circ}$ erhitzt. Nach drei Tagen wurde die gebildete Fumarsäure filtrirt und titrirt.

\begin{tabular}{|c|c|c|c|}
\hline . 12 & 9 & 6 & 3 Stunden \\
\hline $\operatorname{con} 1 / 1.0 \mathrm{~N}$ & $10 \cdot 2$ & $8 \cdot 5$ & $4 \cdot 6$ \\
\hline Fumarsäure gebildet in Procenten. & $23 \cdot 6$ & $19 \cdot 7$ & $10 \cdot 6$ \\
\hline
\end{tabular}

Die Umwandlung nimmt also bei $135^{\circ}$ in $5^{\%}$ iger Lösung lald ein Maximum an, das sich dann nicht mehr ändert. 
Bei derselben Temperatur aber grösserer Concentration wird das Maximum schwieriger erreicht und der Procentzahl nach geändert.

3 Röhren mit je $5 \mathrm{~cm}^{3} 25 \%$ Maleïnsäurelösung, deren Titre genan bestimmt war, warden 9,6 und 3 Stunden auf $135^{\circ}$ erhitzt, nach mehrtägigem Stehen der Titre der Flüssigkeit ober der abgeschiedenen Fumarsäure ermittelt und aus der Differenz der Titrationen die Umwandlung berechnet. Titre der ursprünglichen Lösung: $42 \cdot 16 \mathrm{~cm}^{3} 1 / 10 \mathrm{Na} 0 \mathrm{H}$.

Dauer des Erhitzens . . . . . . . . . . . $96 \quad 6 \quad 3$ Stmden

Titre nach dem Erhitzen ........23.10 $26 \cdot 7 \quad 32 \cdot 1 \mathrm{~cm}^{3} 1 / 10 \mathrm{NaOH}$

Fumarsäure gebildet in Procenten... $46 \cdot 6 \quad 38 \cdot 0 \cdot 25 \cdot 7$

Bei höherer Temperatur tritt bei fortdauerndem Erhitzen eine Abnahme der anfänglich entstandenen Fumarsäure ein.

Je $5 \mathrm{~cm}^{3} 25^{\%}$ Lösung 9,6, 3 Stunden auf $180-190^{\circ}$ erhitzt, Titre der ursprünglichen Lösung: $42 \cdot 16 \mathrm{~cm}^{3} 1 / 10 \mathrm{NaOH}$.

Daver des Erhitzens . . . . $90 \quad 9 \quad 6 \quad 3$ Stunden

Titre nach dem Erhitzen:

Versuch $a \ldots \ldots \ldots \ldots 29 \cdot 0 \quad 28 \cdot 4 \quad 25 \cdot 3 \mathrm{~cm}^{3}$

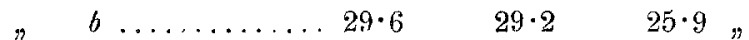

Fumarsäure gebildet in Procenten:

Versuch $a \ldots \ldots \ldots \ldots .32 \cdot 7 \quad 34 \cdot 1 \quad 41 \cdot 3$

$\begin{array}{lllll}b \ldots \ldots \ldots & 29 \cdot 7 & 31 \cdot 2 & 38 \cdot 5\end{array}$

Anch bei sehr hoher Temperatur ist die Umwandlung nie vollständig.

2 Röhren mit je $5 \mathrm{~cm}^{3} 25 \%$ Maleünsäure 4 Stunden auf $240^{\circ}$ erhitzt (jedes in einer besonderen Operation) zeigten eine Umwandlung von $48 \cdot 9$, beziehungsweise $47 \cdot 1 \%$.

Endlich sind noch gleichzeitig wechselnde Concentrationen gleicher Temperatur ausgesetzt worden.

3 Röhren 3 Stunden auf $135^{\circ}$ :

1 enthielt $1 \mathrm{~cm}^{3} 25 \%$ Maleïnsäure, $3 \mathrm{~cm}^{3}$ Fumarwasser,

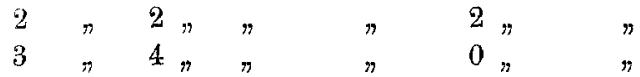

Die abgeschiedene Fumarsäure wurde filtrirt, dann titrirt.

$\begin{array}{lccc} & 1 . & 2 . & 3 . \\ \left.c m^{3} 1\right\}_{10} \text { Na OH verbraucht ...... } 4 \cdot 6 & 19 \cdot 2 & 52 \cdot 1 \\ \text { Fumarsäure in Procenten...... } 10 \cdot 6 & 22 \cdot 2 & 30 \cdot 2\end{array}$


Zunehmende Concentration ist also der Umwandlung günstig.

Wie schon erwähnt, ist eine vollständige Überführung der Maleïnsäure in Fumarsäure durch Erhitzen mit Wasser nicht möglich, und die entgegenstehende Angabe von Tanatar ${ }^{1}$ beruht, auf einem Irrthum.

Der nicht umgewandelte Theil ist aber auch nicht bloss Maleïnsäure, sondern bei höherer Temperatur (iiber $170^{\circ}$ ) ausschliesslich Äpfelsäure, bei niederer ein Gemenge dieser mit Maleïnsäure.

Bei niederen Temperaturen stellt sich demnach ein Gleichgewichtszustand zwischen allen drei Säuren, bei höheren bloss z.wischen Äpfelsäure und Fumarsäure ein.

Um über den ersteren einen annähernden Aufsehluss zu erhalten, wurden $5 \mathrm{~cm}^{3}$ Maleïnsäure von $25 \%$ mit $20 \mathrm{~cm}^{3}$ Fumarwasser vermischt, der Titre bestimmt $(9 \cdot 081 / 10 \mathrm{NaOH})$, dann $23 \mathrm{~cm}^{3} 4$ Stunden auf $140^{\circ}$ erhitzt, nach dem Ausfallen der Fumarsäure der Titre wieder bestimmt $\left(7 \cdot 20 \mathrm{~cm}^{3}\right), 18 \mathrm{~cm}^{3}$ der Lösung mit Kupfercarbonat gesättigt, wiederholt eingedampft und das schliesslich resultirende sehr leicht lösliche Kupfersalz mit Schwefelwasserstoff zerlegt. Das Filtrat yom Schwefelkupfer dunstete zu einer Krystallisation von den Figenschaften der Äpfelsänre ein, das Schwefelkupfer lieferte $0.063 g \mathrm{CuO}$.

Ans diesen Daten berechnet sich die Bildung von Fumarsäure zn $20 \cdot 6 \%$, die der Äpfelsäure zu $14 \cdot 6 \%$ der ursprünglichen Maleïnsäure.

Zur Beurtheilung dieser Verhältnisse ist die Thatsache von Wichtigkeit, dass auch Fumarsäure, mit reinem Wasser erhitzt, in Äpfelsäure übergeht, dass bei höherer Temperatur $\left(170^{\circ}\right)$ neben dieser Maleïnsäure in nachweisbaren Mengen nicht entsteht, und dass dieser Übergang viel schwieriger erfolgt als die Bildung von Äpfelsäure aus Maleïnsäure. ${ }^{2}$

Loydl hat vor längerer Zeit die aus Fumarsäure beim Erwärmen mit Natronlauge entstehende Äpfelsäure untersucht

1 Chem. Centralblatt, 1891, S. 17.

2 Die Äpfeisäure ist dureh das leicht lossliche Kupfersalz von den anderen zwei Säuren leicht zu trennen. Auf Maleïnsäure wurde meist derart gesucht, dass die Mutterlaugen der Fumarsäure mit Kupfercarbonat gesättigt, und die direct und beim Eindampfen ausfallenden schwer löslichen Cu-Salze 
und die auffallende Beobachtung gemacht, dass diese beim Erhitzen ansschliesslich in Fumarsäure übergeht und gar keine Maleînsäure bildet.

Um. genauer festzustellen, ob die Äpfelsäure aus Maleïnsäure dasselbe oder vielleicht das entgegengesetzte Verhalten zeigt, babe ich aus beiden Sänren, und zwar sowohl durch Erhitzen mit Wasser, als mit Natronlauge die Äpfelsäure in grösseren Mengen dargestellt.

Äpfelsäure aus Malernsäure. Maleïnsäure wurde in $25 \%$ Lösung 4 Stunden auf $180-190^{\circ}$ erhitzt, nach dem Erkalten die Lösung von der im Rohre abgeschiedenen Fumarsâure getrennt, eingedampft, die Krystallisation durch Lösen in möglichst wenig Wasser von Fumarsäure befreit, wieder eingedampft und dies so lange wiederholt, bis die Krystalle in Fumarwasser vollkommen klar auflöslich sind. Dureh nochmaliges Umkrystallisiren erhält man die Säure dann ganz rein.

Sie krystallisint in meist za Rosetten vereinigten ziemlich deutlichen Prismen, ist so gut wie picht hygroskopisch und krystailisirt zum Unterschiede von der activen Äpfelsäure leicht beim Verdunsten an freier Luft. In Wasser ist sie auch merklich schwerer löslich als die active Säure. Sie schmilzt bei $130-131^{\circ}$. Zur Analyse wurde sie über Schwefelsäure getrocknet.

Die Bestimmungen verschiedener Darstellungen und Fractionen ergaben:

1. $0 \cdot 1768 \mathrm{~g}$ lieferten $0.2318 \mathrm{~g} \mathrm{CO}_{2}$ und $0.0722 \mathrm{~g} \mathrm{H}_{2} \mathrm{O}$.

2. $0.2098 \mathrm{~g} " 0.2757 \mathrm{~g}$ " $0.0850 \mathrm{~g}$ "

3. $0.1801 \mathrm{~g} " 0.2381 \mathrm{~g} "$ " $0.0750 \mathrm{~g}$ "

$\begin{array}{crrrr} & 1 . & 2 . & 3 . & \underbrace{\text { Berechnet }} \\ \mathrm{C} \ldots \ldots \ldots & 35 \cdot 81 & 35 \cdot 84 & 36 \cdot 05 & 35 \cdot 81 \\ \mathrm{H} \ldots \ldots \ldots & 4.46 & 4.50 & 4.62 & 4.47\end{array}$

Analyse 2 ist mit der ersten, 3 mit der fünften Fraction einer Darstellung ausgefuhrt, so dass sicher steht, dass andere leicht

untersucht wurden. Da das maleïnsaure Cu äusserst charakteristisch ist und viel schwieriger ausfällt als das Kupfersalz der Fumarsäure, kaun man dasselbe nicht übersehen. Überdies habe ich meist die schwer löslichen CuSalze mit $\mathrm{SH}_{2}$ zerlegt eingedampft und die Sätre untersucht. Sind grössere Mengen von Maleînsäure neben Fumar- und Äpfelsäure in Lösung, schiessen sie beí abgestuftem Eindampfen in den mittleren Fractionen aus. 
lösliche Producte als Äpfelsäure in nennenswerther Menge nicht entstehen.

Diese Äpfelsäure sollte optisch nicht activ sein. Die isolirte Säure ist in dieser Richtung zwar nicht, dafür aber die iiber der abgeschiedenen Fumarsäure stehende Reactionsflissigkeit untersucht worden, welche nach der Titration etwa $16 \%$ Äpfelsäure hielt. In einem Falle, wo $25 \mathrm{~g}$ chemisch reiner Maleïnsäure mit $100 \mathrm{~g}$ Wasser 6 Stunden auf $180-190^{\circ}$ erhitzt worden waren, war im Apparate von Lippich im $300 \mathrm{~mm}$-Rohr die Ablenkung thatsächlich Null, da im Mittel die optische Neutralität sich mit reinem Wasser und mit der Lösung bei $1 \% 484$ einstellte. Bei einem zweiten Versuch, der sonst ganz wie der vorige, nur mit käuflicher Maleïnsäure angestellt war, zeigte sich eine kleine, aber doch merkliche Differenz, wie sie freilich bloss mit einem so ausserordentlich feinen Instrument, wie das ron Lippich construirte, erkennbar ist.

Die Ablesungen mit der Lösung ergaben $1^{\circ} 554$, mit Wasser $1^{\circ} 492$, wie ausser mir auch Herr Dr. P. Czermak gefunden hat, dem ich für diese Beibilfe auch hier danke.

Da ich den Gegenstand nicht weiter verfolgt habe, enthalte ich mich weiterer Schlïsse.

Die Überführung der Malë̈säure in Äpfelsäure mit Natronlauge gelingt leicht, wenn man die von Loydl für die Darstellung der inactiven Äpfelsäure aus Fumarsäure gegebene Vorschrift anwendet. Zweckmässiger als das Extrahiren mit $\ddot{A}$ ther ist es, die mit der berechneten Menge Schwefelsäure versetzte Lösung durch zweimaliges starkes Eindampfen von der Hauptmenge des Glaubersalzes zu befreien und die letzte Mutterlange mit überschüssigem Alkohol zu vermischen. Die yon den letzten Resten Natriumsulfat getrennte alkoholische Flüssigkeit wird nach dem Eindampfen in derselben Art verarbeitet, wie vorher beschrieben ist. Neben Äpfelsäure sind nicht unerhebliche Mengen von Fumarsäure entstanden. Die so gewonnene Äpfelsäure unterscheidet sich von der durch Erhitzen mit Wasser dargestellten bloss dadurch, dass die zuerst anschiessenden Fractionen etwas höher schmelzen, sie verflüssigen sich vollständig erst bei $140^{\circ}$; die späteren schmelzen glatt bei $130-131^{\circ}$. Möglicherweise ist 
den schwerer löslichen Antheilen etwas Fumar- oder Maleünsäure beigemischt.

Äpfelsäure aus Fumarsäure babe ich nach der Vorschrift von Loydl und dorch Erhitzen der Fumarsäure mit Wasser dargestellt. Die Umwanllung in letzterer Art geht, wie schon erwähnt, viel schwieriger als bei der Maleïnsänre vor sich.

Bei folgenden Versuchen ist die Säure entweder mit reinem Wasser oder gesättigter Fumarsäurelösung erhitzt, nach dem völligen Umkrystallisiren der Titre der Lösung bestimmt und aus demselben die Umwandlung berechnet worden.

Umgewandelt:

$2.805 g$ Fumarsäure mit $30 \mathrm{~cm}^{3} 31 / 2$ Stunden auf $135^{\circ}$. $0.8 \%$.

$5 \mathrm{~g} \quad n, \quad n 10 \mathrm{~cm}^{3} \quad$ " $\quad n \quad n \quad 1 \cdot 1$

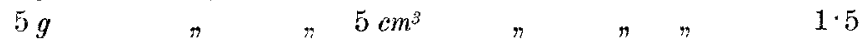

$10 \mathrm{~g} \quad " \quad$ " $50 \mathrm{~cm}^{3} 6$ Stunden a af $180-190^{\circ} .11 \cdot 6$

$10 \mathrm{~g} \quad \# \quad \# 10 \mathrm{~cm}^{3} 5 \quad \# \quad, 170 . \quad 18 \cdot 71$

Die Limwandlung ist bei höherer Temperatur grösser, sie scheint durch grössere Wassermengen beeinträchtigt zu werden.

Die isolirte und gereinigte Äpfelsäure zeigte in Löslichkeit, Krystallform und Schmelzpunkt keinerlei Unterschied mit der aus Maleïnsäure, dasselbe gilt von jener, die nach Loydl vermittelst Ätznatron gewonnen worden ist. Die mit reinem Wasser dargestellte wurde analysirt.

$0 \cdot 1594 \mathrm{~g}$ gaben $0 \cdot 2088 g \mathrm{CO}_{2}$ und $0.0659 \mathrm{~g} \mathrm{H}_{2} \mathrm{O}$.

C $\ldots . .35 \cdot 81$
$\begin{aligned} & \text { Berechnet } \\ & \text { für } \mathrm{C}_{4} \mathrm{H}_{6} \mathrm{O}_{5}\end{aligned}$$\quad \begin{array}{r}35 \cdot 95 \\ 4 \cdot 65\end{array}$

Loydl hat von seiner inactiven Äpfelsäure mitgetheilt, dass sie, auf $200^{\circ}$ erhitzt, ausschliesslich Fumarsäure liefert. Da die gewöhnliche active Säure bekanntlich bei höherer T'emperatur

1 Die von der unveränderten Fumarsäure getrennte Lösung ist mit Kupfercarbonat gesättigt und eingedampft worden. Die charakteristischen Krystalle des maleïnsauren Kupfers treten nicht auf. Die beim Eindampfen erhaltenen Abscheidungen mit Schwefelwasserstoff zersetzt, lieferten wieder nur Fumarsäure und kleine Mengen einer syrupösen Lange, aus der immer noch Fumarsäure, nicht aber Maleïnsäure zu gewinnen war. 
in Fumar- und Maleïnsäure übergeht, würde eine solche Verschicdenheit von grossem und besonders stereochemischem Interesse sein. Die Angaben von Loydl beruhen aber auf einem Irrthum.

Sowohl die active Äpfelsäure, als auch die beiden inactiven aus Maleïnsäure und Fumarsäure, verhalten sich beim Erhitzen ganz gleich. Bei raschem Erhitzen auf $200^{\circ}$ und wenn die flitchtigen Zersetzungsproducte im Vacuum oder durch einen Luftstrom weggeführt werden, liefern sie ganz gleichmässig annählernd gleiche Mengen Maleïnsäure und Fumarsäure, letztere itberwiegt aber bedeutend (etwa $9 \mathrm{mal}$ ), wenn die Temperatur langsam ansteigt daher Maleïnsänreanhydrid, sowie das Wasser in das heisse Bad zurïckfliessen können. Wahrscheinlich hat Loydl, der seinen Versnch nicht genauer beschreibt, lie letztgenannten Bedingungen eingehalten, vielleicht sogar im geschlossenen Rohr erhitzt.

Ein wesentlicher Unterschied besteht also zwischen der inactiven Äpfelsänre aus Maleönsäure und aus Fumarsäure nicht.

Dabei ist allerdings ein Umstand zu berücksichtigen. Da die Fumarsäure bei niedrigerer Temperatur schwierig, bei erhöhter Temperatur aber leichter in Äpfelsäure ïbergeht, ist wahrscheinlich der Äpfelsäure, die durch Erhitzen wässeriger Maleïnsäurelösung auf $180-190^{\circ}$ entstanden ist, solche beigemischt, die aus der primär gebildeten Fumarsäure unter Aufnahme von Wasser sich bildet. Dafur spricht vor Allem der Umstand, dass bei höberen Temperaturen $\left(170^{\circ}\right)$ bei fortschreitendem Erbitzen die Fumarsäure abnimmt, daher in Äpfelsäure iibergeht.

Wie Maleïnsäure sich beim Erhitzen für sich verhält, ergaben folgende Versuche:

3 Röhren mit je $1 \mathrm{~g}$ Maleïnsäure wurden auf $130^{\circ}$ erhitzt, und zwar in der Dauer von 3, 6 and 9 Stunden.

Nach dem Erkalten war Fumarsäure in grossen Krystallen abgeschieden. In jedes Rohr kam $5 \mathrm{~cm}^{3}$ Fumärwasser, dann wurde erwärmt, wobei kein Schmelzen eintrat, was auf die Abwesenheit von. Maleïnsäureanhydrid schliessen lässt. Die erkalteten Lösungen wurderi nach 18 Stunden titrirt.

$\begin{array}{llll}\text { Dauer des Erhitzens . . . } & 9 & 6 & \text { 3 Stundeu } \\ \text { Titre der Lösung ...... } & 5 \cdot 75 & 9 \cdot 82 & 18 \cdot 1 \mathrm{~cm}^{3} 1 / 10 \mathrm{Na} 0 \mathrm{H} \\ \text { Fumarsäure gebildet } \ldots & 88 & 77 & 58 \%\end{array}$


Die Lmwandlung geht demnach anfänglich rasch vor sich und nimmt später nur langsam zt. Die Lösungen, die drei und 9 Stunden erhitzt waren, wurden mit Kupfercarbonat neutralisirt und eingedampft. Die erstere schied reichlich, die letrtere fast gar kein maleïnsaures Kupfer ab, beide gaben ein nicht krystallisirtes sehr leicht lösliches Salz, offenbar das der Äpfelsäure, das auch, mit Schwefelwasserstoff zerlegt, die der inactiven Äpfelsäure charakteristische Form zeigte.

Die Äpfelsäure entsteht demnach beim Erhitzen der Malënsäure für sich oder mit Wasser unter allen Umständen. Der Übergang von Maleïnsäure in Fumarsäure wäre desshalb derart erklärbar: aus Wasser und Maleïnsäure entstebt zunäehst Äpfelsäure, die dann nuter Wiederabspaltung von Wasser in Fumarsäure tibergeht.

Dass die Äpfelsäure, in wässeriger Lösung erbitzt, unter Unständen, d. b. bei starker Concentration in Fumarsäure thatsächlich ubbergeht, wie ich gefunden babe, kann natülich nicht iiberraschen. Bei geringeren Concentrationen tritt dieser Übergang aber gar nicht ein.

Eine 25\% Lösung von Äpfelsäure (aus Maleïsäure dargestellt) in Wasser wurde mit Fumarsäure gesättigt und mit Fumarwasser in folgenden Verhällnissen vermischt.

$\begin{array}{cc}\text { Aptelsäure } & \text { Fumarwasser } \\ 5 & 0 \\ 4 & 1 \\ 3 & 2 \\ 2 & 3 \\ 1 & 4\end{array}$

Die Röhren wurden $3 \frac{1}{2}$ Stunden aut $140^{\circ}$ erhitzt, zeigten aber mit Ausnahme des ersten, welches nach wochenlangem Stehen einige winzige Kryställchen ansetzte, nicht die geringste Abscheidung.

Nicht anders verliefen Versuche mit der activen Äpfelsäure.

Wenn also selbst eine $20 \%$ ige Apfelsäurelösung keine Fumarsänre liefert, so kann noch viel weniger die Äpfelsäure, welche beim Erhitzen einer $5 \%$ igen Lösung von Maleïnsüure entstebt, in Fumarsäure übergehen, und darum kommt die Äpfelsäure als Zwischenglied der Umlagerung nicht in Betracht. Da aber nachweisbar Äpfelsäure immel entsteht, wenn die Umlagerung zu beobachten ist, also beide Processe parallel laufen, bleibt kein 
anderer Ausweg, als die Annahme, die Bildung ron Äpfelsäure begiinstige katalytisch die Umwandlung der Malcïnsäure in Fımarsänre. Auf Grund dieser Annahme lassen sich die mitgotbeilten Thatsachen einheitlich erklären, anf Grund derselben ist auch eine Vorstellung über das Verhalten der Äpfelsäure beim Erhitzen zu gewinnen. Da umso weniger Fumarsäure grebildet wird, je rascher man die Destillationsproducte wegschafft, so dürfte die Äpfelsäure unter Wasserabspaltung wahrscheinlich nur Maleïnsäure, beziebungsweise deren Anlydrid geben, die aber, mit Wasser in Beruhlung, sich so verändert wie beim Erlitzen im geschlossenen Rohr. Beim Erhitzen kleiner Mengen von Äpfelsäure im Reagirrohr kann man unter Umständen fast nur Maleïnsäure erhalted.

Wahrscheinlich wird auch die Bildung von Fumarsäure beim Erhitzen fester Malerinsäure in derselben Art erfolgen, da ja auch hier anfänglich Maleünsäureanhydrid und W'asser entstehen müssen, welch' letzteres auf unveränderte Säure einwirken wird.

\section{Einwirknng von Sänren auf Maleïnsäure.}

Schon Kekulé hat gefunden, dass Chlor-, Brom- und Jodwasserstoffsäure, sowie Salpetersäure die Maleinsäure in Fumarsäure nmlagern. Dieselbe Wirkung fand ich bei Schwefel-, Phosphor-Phosphoriger- und Arsensäure, sowie bei verschiedenen organischen Säuren.

Die Unwandlung durch starke anorganische Süuren ist von der Concentration derselben abhängig, bei den Halogenwasserstoffsäurea und der Salpetersäure ist jene der Concentration direct proportional, bei der Schwefelsäure ist eive bestimmte mittlere Concentration am günstigsten.

Von den Halogenwasserstoffsäuren wandelt Jodwasserstoffsäure am raschesten, Bromwasserstoffsäure am schwächsten um. In qualitativer Beziehnng ist von Wichtigkeit, dass neben Fumarsäure stets Additionsproducte der Maleïnsäure entstehen.

Von Fittig ist bereits vor langer Zeit nachgewiesen worden, dass ratchende Bromwasserstoffsäure die Maleïnsäure in eine Verbindung überführt, die nach ihm möglicherweise ein gemischtes Anhydrid von Fumarsäure und Brombernsteinsäure sein könnte, da sie, mit Wasser behandelt, Fumarsäure hinterlässt, während 
Brombernsteinsäure in Lösung geht und einen der vermutheten Zusammensetzung nahe liegenden Bromgehalt besitzt.

Diese Beobachtung ist merkwürdigerweise späterhin nicht weiter beachtet worden, obzwar sie beweist, dass der Bildung von Fumarsäure die von Brombernsteinsäure parallel verlaufen muss, da die Fumarsäure Bromwasserstoff äusserst schwierig. aufnimmt.

Ganz dasselbe konnte ich für die Einwirkung von Salzsäure nachweisen.

$5 \mathrm{~g}$ Maleïnsäure in einem Stöpselcylinder mit $25 \mathrm{~cm}^{3}$ bei $0^{\circ}$ gesättigter Salzsäure übergossen, gingen, ohne sich merklich zu lösen, im Verlauf von 4 Tagen in ein weisses Pulver uber vom Aussehen der Fumarsäure. Dasselbe wurde vor der Pumpe gesammelt, mit wenig Salzsäure gewaschen und über Ätzkali getrocknet, bis es nicht mehr nach Salzsäure roch. Es wog dann $4 \cdot 34 g$ und war stark chlorhältig.

$0.2098 \mathrm{~g}$, mit Natriumamalgam zerlegt, gaben $0.0905 \mathrm{~g} \mathrm{AgCl}$.

Diesem Chlorgehalt entsprechen $0.0962 g$ Chlorbernsteinsäure, das Pulver enthielte darnach $45 \cdot 8 \%$ Chlorbernsteinsäure; eine Mischnng von je 1 Mol. dieser und 1 Mol. Fumarsäure hätte einen Chlorgehalt von $56 \cdot 7 \%$.

Das Pulver mit wenig kaltem Wasser extrahirt, hinterliess Fumarsäure, während ein grosser Theil in Lösung ging, der, ubber Schwefelsäure abgedunstet, zuerst noch etwas Fumarsäure abschied und dann giänzende, in Wasser leicht lösliche Krystalle, die sebr stark chlorhältig sind, bei $151-152^{\circ}$ schmelzen, demnach zweifellos Chlorbernsteinsäure sind. Ganz ähnliche Krystallisationen gaben das ron dem ursprïnglichen Pulver getrennte Filtrat, sowie die zum Waschen benitzte Salzsäure.

Bei Einwirkung concentrirter Salzsäure auf Maleïnsäure entsteht also neben Fumar'säure Chlorbernsteinsäure, und letztere gewiss nicht aus primär gebildeter Fumarsäure, wie folgender Versuch lehrt.

In mit Kalk verscblossenen Cylindern wurden je $2 g$ Fumarsäure mit $10 \mathrm{~cm}^{\mathrm{s}}$ Salzsäure übergossen, einmal mit concentrirter, das anderemal mit einem Gemisch von gleichem Volum Säure und Wasser; in zwei andere Cylinder kamen die zwei Salzsäuren 
für sich, und von Zeit zu Zeit wurde der Titre acidimetrisch und der Salzsäuregehalt nach Volhard ermittelt.

\begin{tabular}{|c|c|c|c|c|}
\hline & dem gle & $\begin{array}{l}\text { ure mit } \\
\text { Volum Wasser }\end{array}$ & Concen & te Salzsäure \\
\hline & Je $1 \mathrm{~cm}^{3}$ & rauchte $\mathrm{cm}^{3} 1 / 10$ & $\mathrm{Je} 1 \mathrm{~cm}^{3} \mathrm{~V}$ & auchte $\mathrm{cm}^{3} 1 / 10$ \\
\hline $\mathrm{Am}$ & $\mathrm{NaOH}$ & Silberlösung & $\mathrm{NaOH}$ & Silberlösung \\
\hline$=4 / 3^{1}$ & $57 \cdot 3$ & $57 \cdot 3$ & $113 \cdot 8$ & $113 \cdot 5$ \\
\hline$=0$ & $57 \cdot 3$ & $57 \cdot 0$ & $113 \cdot 7$ & $113 \cdot 4$ \\
\hline 涪 & $57 \cdot 3$ & $57 \cdot 1$ & $113 \cdot 9$ & $113 \cdot 7$ \\
\hline$(4 / 3$ & $57 \cdot 3$ & $56 \cdot 4$ & $113 \cdot 8$ & $113 \cdot 5$ \\
\hline 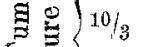 & $57 \cdot 4$ & $56 \cdot 2$ & $113 \cdot 8$ & $113 \cdot 5$ \\
\hline$\underbrace{2}$ & $\left\{\begin{array}{l}57 \cdot 9 \\
57 \cdot 3\end{array}\right.$ & $\begin{array}{l}56 \cdot 8 \\
57 \cdot 1\end{array}$ & $114 \cdot 0$ & $113 \cdot 1$ \\
\hline
\end{tabular}

Weder der Gesammttitre, noch der Gehalt an Salzsäure änderte sich, demnach entsteht unter diesen Umständen aus Fumarsäure Chlorbernsteinsäure nicht.

Nachdem hiedureh constatirt war, dass Salzsäure auch bei grosser Concentration auf Fumarsäure ohne Wirkung ist und überdies sehr concentrirte Salzsäure, in gut verkorkten Gefässen längere Zeit aufbewahrt, durch Verdunstung den Titre nicht ändert, habe ich den Nachweis versucht, ob auch bei geringeren Concentrationen der Salzsäure Maleïnsäure in Fumarsäure übergeht und daneben stets Chlorbernsteinsäure gebildet wird.

Zu diesem Behufe wurden 25\% Maleïnsäurelösung, Wasser und concentrirte Salzsäure mit Fumarsäure gesättigt und in folgendem Verhältniss gemischt:

Lösung der

Nr. Maleïnsăme

$\begin{array}{rr}1 & 8 \\ 2 & 4 \\ 3 & 2 \\ 4 & 6 \\ 5 & 4 \\ 6 & 2 \\ 7 & 4 \\ 8 & 2 \\ 9 & 2 \\ 10 & 1\end{array}$

Titre berechnet Wasser Salzsäure $\mathrm{cm}^{3} 1 / 10 \mathrm{NaOH} \mathrm{cm}^{31} / 10 \mathrm{AgNO}_{3}$

-
4
6
-
2
4
-
2
-
-

$57 \cdot 4$

$40 \cdot 6$

$32 \cdot 3$

$72 \cdot 2$

$63 \cdot 8$

$55 \cdot 4$

$86 \cdot 9$

$78 \cdot 5$

$101 \cdot 7$

$1.09 \cdot 8$
$23 \cdot 3$

$23 \cdot 3$

$23 \cdot 3$

$46 \cdot 6$

$46 \cdot 6$

$46 \cdot 6$

$69 \cdot 8$

$69 \cdot 8$

$93 \cdot 1$

$104 \cdot 8$

1 Tag der Mischung. 
Die obiger Berechnung za Grunde gelegten Titres waren fur die mit Fumarsäure gesättigten Lösungen: Maleïnsäure $42 \cdot 7$, Wasser 0.8 , Salzsăure $116.4 \mathrm{~cm}^{3} 1 / 10 \mathrm{NaOH}$.

Die Abscheidung ans den gemischten Lösungen erfolgte in alleu Röhren schon nach 1 Stunde, sie war in jenen mit böheren Ziffern entsprechend der Zahlenordnung grösser, in 4 und 7 war unverkennbar neben Fumarsäure sehr viel Maleïnsäure ausgefallen, die in den späteren Tagen in Lösung ging. Hiedurch erklärt sich die später beobachtete Zunalıme des Titres in 4 und 7. Die Abscheidung nahm wäbrend der Versucbszeit zu, umsomehr, je höher die Röhrenziffern waren.

Folgende Tabelle entlält die Werthe, welche im Verlaufe dreier Wochen durch Titration mit $1 / 10 \mathrm{NaOH}$ und $1 / 10$ Silberlösung hervorgingen.

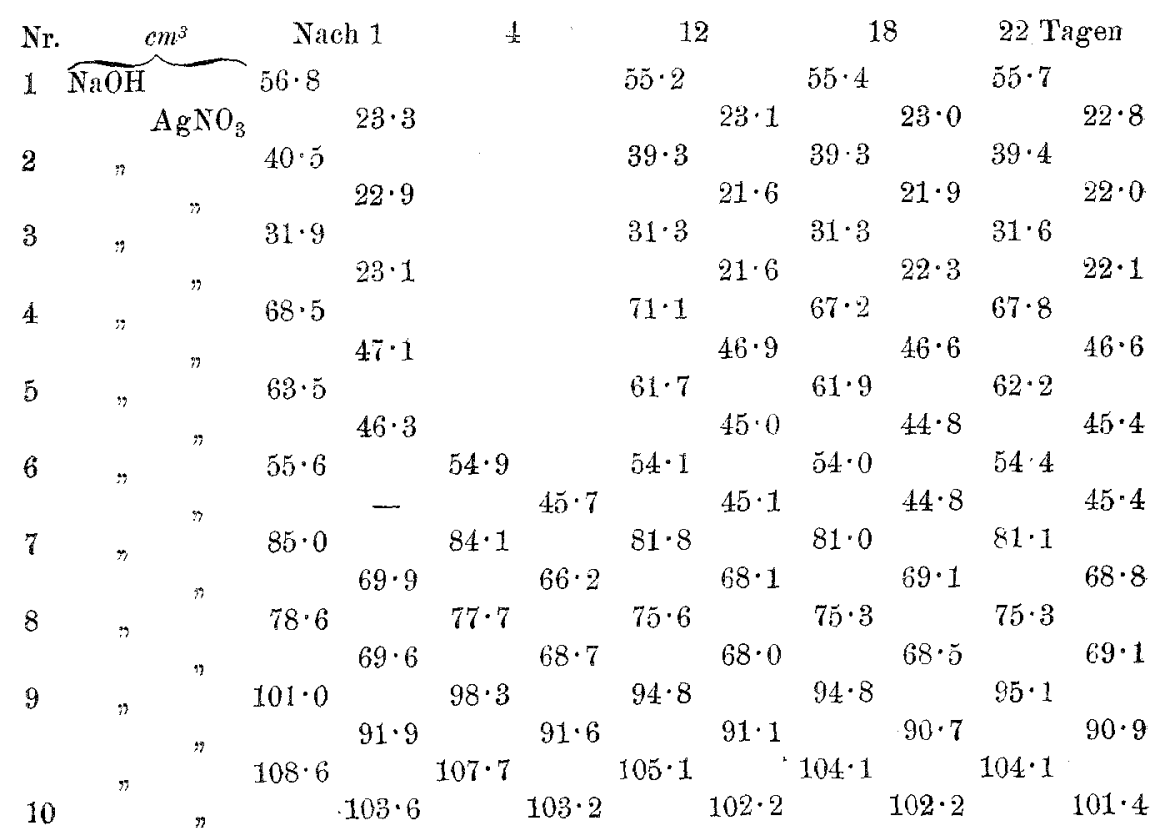

Die Werthe zejgen geringe Unregelmässigkeiten, die sich leicht erklären, da zu jeder Titration Bruchtheile eines Cubikcentimeters in Verwendung kamen, lassen aber zweifellos erkennen, dass nicht nur rasch eine Verminderung der berechneten Werthe, sondern auch später neben der Abnahme des 
Gesamnattitres eine Abnahme der Salzsäure stattgefunden hat; beide Abnahmen sind umso merklicher, je günstiger die Bedingungen für die Bildung von Chlorbernsteinsäure sind, je mehr Salzsänre bei gleichem Volum vorhanden ist.

Die Entstehung der Fumarsäure ist also unter allen Umständen an die der Chlorbernsteinsänre gebunden, und es ist mehr denn wahrscheinlich, dass nach dem wichtigen Versuch von Fittig Ähnliches für die Einwirkung der Bromwasserstoffsäure auch bei grösserer Verdünnung gelten wird.

Jodwasserstoff \& üu re bewirkt die Unsetzung der Maleïnsäure in Fumarsäure sehr rasch. $1 \mathrm{~g}$ Maleïnsäure mit $5 \mathrm{~g}$ Jodwasserstoffsäure vom sp. G. 1.96 ibergossen und nach 1 Tage auf eine poröse Platte gebracht, wog, über Kalk getrocknet, $0.86_{z} g$, mit $3 \mathrm{~cm}^{3}$ Wasser ïbergossen, abgesangt und mit Fumarwasser gewasehen, nur mehr $0 \cdot 71 \mathrm{~g}$. Die wässerige Lösung enthielt so gut wie kein Jod, hinterliess aber Kryställchen, die Ähnlichkeit mit Bernsteinsäure hatten.

$3 g$ Maleïnsäure mit $5 g \mathrm{JH}$ gaben $2 \cdot 4 g$ in Fumarwasser unlösliches Product, also iiber $70 \%$. Auch hier war in den verschiedenen Filtraten selbst nach dem vorsichtigsten Eindunsten ein Körper von den vermutheten Eigenschaften der Jodbernsteinsänre in irgend nennenswerther Menge nicht nachweisbar.

Jodbernsteinsäure durch Erhitzen von Fumarsäure mit einer bei - $20^{\circ}$ gesättigten Lösung von Jodwasserstoffsäure in Eisessig durch mehrere Stunden auf Wasserbadtemperatur dartustellen, gelang nicht, nur Bernsteinsäure war nachweisbar, ausserdem entstanden Gase.

De Jodbernsteinsäure ist also gewiss sehr unbeständig; und in Folge dessen wäre die Erklärung von Wi slicenus, wie Malënsäure dureh Jod wasserstoff in Fumarsäure übergeht, mit den Thatsachen nicht im Widerspruche.

Nachdem es mir aber in besonderen Versuchen gelungen ist, in der abgeschiedenen Fumarsäure jedesmal Bernsteinsäure vom richtigen Schmelzpunkt und allen sonstigen Eigenschaften nachznweisen, balte ich dafür, dass der Vorgang auch hier anders verläuft. Da. Malë̈nsäure mit Jodwasserstoff stets und friher Jod abscheidet, ehe Fumarsäure ausfällt, während Jodwasserstoffsäure derselben Concentration unter gleichen Umständen 
ungefärbt bleibt und die Menge des ausgeschiedenen Jods weiter unzweifelhaft umso grösser ist, je mehr Fumarsäure gebildet wurde, rührt die Jodabscheidung gewiss von der Bildung der Bernsteinsäure her, und diese entsteht nicht später, wie die Fumarsåıre. Letztere wird, analog wie früher anseinandergesetzt ist, in dem Masse entstehen, wie ein Theil der Maleïnsäure in Jodbernsteinsäure und diese weiter in Bernsteinsäure ilbergefuburt wird.

Dass Salpetersäure die Maleïnsäure umlagert, hat schnn Kekulé angegeben. Die Umwandlung erfolgt aber nicht so leicht, wie man aus der knappen Angabe wörtlich entnehmen könnte. Nach kurzem Kochen von Maleïnsäure in Substanz oder coneentrirter wässeriger Lösnng mit nicht zu verdünnter Salpetersäure scheiden sich zwar Krystallkörner ab, die aber nichts Anderes als unveränderte Säure sind, und bei Zimmertemperatur erfolgt die Umwandlung äusserst träge.

Nahezu vollständig geht sie beim Eindampfen vor sich.

万ิ $\mathrm{cm}^{3}$ einer $25 \%$ Maleïnsäurelösung mit $1 \mathrm{~cm}^{3} \mathrm{NO}_{3} \mathrm{H}(\mathrm{sp} . \mathrm{G} .1 \cdot 40) \mathrm{am}$ Wasserbad eingedampft, mit Wasser nochmals zur Trockne gebracht und dann mit Fumarsäure gewaschen hinterliessen Fumarsäure, die mit $1 / 10 \mathrm{NaOH}$ titrirt, $159 \cdot 3 \mathrm{~cm}^{3}$ brauchte. Das entspricht $0.924 \mathrm{~g}$ Fumarsänre, also einer Umwandlung von $92 \%$. Das Filtrat enthielt nicht die Spur Oxalsäure.

Den Grund dieser Zersetzung anzugeben vermag ich nicht. Er dürfte in der Bildung von nitrosen Gasen kaum liegen, da sich zeigte, dass Maleïnsäure und Kaliumnitrit, in wässeriger Lösung. mit Schwefelsäure vermischt, Fumarsäure nicht bilden. Vielleicht. steht jene mit einer totalen Oxydation der Maleïnsäure im Zusammenhange, wofür spricht, dass bei den später angefübrten Versucben S. 125 deutlich Gasentwickelung zu beobachten war.

Die geringe Wirkung von Schwefelsä ure auf Maleïnsäure bat Kekulé schon hervorgehoben. Dieser Angabe sei noch zugefügt, dass concentrirte und mässig verdünnte Schwefelsäure Maleînsäure sehr schwierig lösen. Da später beschriebene Versuche es nothwendig machten, habe ich $1 \mathrm{~g}$ Maleïnsäure in $100 \mathrm{~g}$ Wasser mit $10 \mathrm{~cm}^{3} 1 /{ }_{10} \mathrm{H}_{2} \mathrm{SO}_{4}$ gelöst, 3 Stunden am Wasserbad erwärmt, dann eingedampft. Der Rïckstand, mit Fumarwasser auf $10 \mathrm{~cm}^{3}$ gebracht, schied auch nach wochenlangem Stehen nicht die Spur Fumarsäure ab, diese ist also nicht entstanden. 
Wie die Versuche auf S. 125 und 127 zeigen, hat Schwefelsäure auch bei grösserer Concentration eine sehr geringe Wirkung, bei höherer Temperatur setzt sie sogar die Wirkung des Wassers herab.

Ganz dasselbe gilt von Benzolsulfonsäure. Oxalsäure vermag bei Wasserbadtemperatur die Maleïnsäure nicht umzusetzen, bei höherer 'Temperatur ist die Umwandlung rascher, als wenn Wasser allein wirkt, dann ist aber auch Zersetzung der Oxalsäure eingetreten und Kohlendioxyd, sowie Ameisensäure entstanden.

Nachstehende Versuche geben Aufsehluss über die relative Geschwindigkeit, mit der die Maleïnsäure unter dem Einfluss verschiedener Säuren in Fumarsäure übergeht.

1. $1 \mathrm{~cm}^{3} 15 \%$ Maleïnsäure vermischt mit $4 \mathrm{~cm}^{3} 4$ fach Normal-Chlorwasserstoff 1), Bromwasserstoff 2), Jodwasserstoffsäure, Schwefel- und Salpetersïmre und in Glasröhren umgeschmolzen. Nach sechs Tagen alles klar, nur in 3 kleine Kryställchen. Dann 1 Stunde im kochenden Wasserbad erhitzt, 18 Stunden stehen gelassen. 4 und 5 klar, in 1, 2, 3 Abscheidungen von Fumarsäure, die durch Titration bestimmt wurden.

$\begin{array}{cccc}\text { Zur Titration verbraucht } & 1 & 2 & 3 \\ \mathrm{~cm}^{3} 1 / 10 \mathrm{~N} \text {. NaOH. ....... } 13 \cdot 3 & 6 \cdot 0 & 35 \cdot 1\end{array}$

Mischungen 1,2 und 3,1 Stunde anf $103^{\circ}$ erhitzt, sonst wie früher.

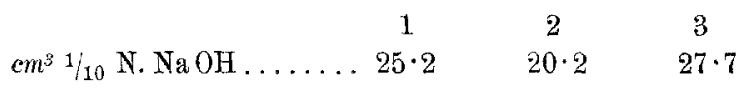

Trotz der etwas differirenden Temperatur hat in beiden Fällen Jodwasserstoff am stärksten, Bromwasserstoff am schwächsten umgewandelt, Salpeter- und Schwefelsäure gar nicht, denn die Röhren blieben auch nach längerem Stehen noch ganz durehsichtig.

2. $1 \mathrm{~cm}^{3}$ Maleïnsäurelösung vermischt mit $10 \mathrm{f}$. N. Schwefels. (a) Salpetersäure (b) Salzsäure (c), und zwar in 1) im Verbältniss 1 Vol. : 5 Vol, in 2) im Verbältniss 1:4:1 Wasser, in 3): 1:3:2 Wasser, in 4): 1:2:3 Wasser.

Die Abscheidung von Fumarsäure erfolgte in den Mischungen mit Salzsäure am frïhesten und reichlichsten. Nach 24 Tagen wurden die oben stehenden Flüssigkeiten mit einer Pipette entfernt, was in Folge der guten Ausbildung der Krystallisation 
leicht war, die Krystalle rasch mit Fumarwasser abgespült, aufs Filter gebracht, gewaschen und titrirt.

\begin{tabular}{cccc}
\multicolumn{5}{c}{ Verbrauch an } & & \\
& $1 / 10 \mathrm{NaOH} \mathrm{cm}^{3}$ & & $\mathrm{em}^{3} 1 / 10 \mathrm{NaOH}$ \\
$1 a$ & $0 \cdot 5$ & $3 a$ & $1 \cdot 4$ \\
$1 b$ & $16 \cdot 7$ & $3 b$ & $3 \cdot 2$ \\
$1 c$ & $26 \cdot 5$ & $3 c$ & $7 \cdot 1$ \\
$2 a$ & $1 \cdot 4$ & $4 a$ & $1 \cdot 1$ \\
$2 b$ & $9 \cdot 9$ & $4 b$ & $0 \cdot 2$ \\
$2 c$ & $23 \cdot 6$ & $4 c$ & $2 \cdot 2$
\end{tabular}

Die Salzsäure wirkt bei allen Verdünnungen am stärksten. Die Salpetersäure bei grosser Concentration stärker wie die Schwefelsäure, bei sehr geringer dagegen schwächer. Die Schwefelsäure hat überhaupt geringen Einfluss, der bei mittlerer Concentration noch am grössten zu sein seheint.

Die Unterschiede in der Wirkung der drei Säuren werden umso kleiner, je mehr sie verduint sind.

3. Im Hinblicke auf die Erfahrungen, die auf anderen Gebieten uber den Einfluss von Salzen anf Reactionsgeschwindigkeiten gemacht worden sind, ersehien es nicht iiberflissig, anch diesen festzustellen.

$4 \mathrm{~cm}^{3} 25 \%$ Maleïnsänre mit $16 \mathrm{~cm}^{3} 10 \mathrm{f}$. N. Salzsäure gemiseht, $6 \mathrm{~cm}^{3}$ in ein Rohr gefüllt 1), der Rest in zwei Hälften getheilt, die eine unter Kühlung mit überschüssigem Lithiumchlorid, die zweite ebenso mit Salmiak vermischt, geschüttelt, rasch filtrirt, und wieder je $6 \mathrm{~cm}^{3} 2$ ) und 3) in ein Rohr gebracht. Die Abscheidung von Fumarsäure erfolgte in 2 am raschesten, am langsamsten in 3, späterhin wurde sie in 2 langsamer als in den anderen Röhren.

Nach 12 Tagen wurde die Fumarsäure getrennt und titrirt.

\begin{tabular}{|c|c|c|c|}
\hline & 1 & 2 & 3 \\
\hline$c m^{3} 1 / 10 \mathrm{NaOH}$ & $20 \cdot 7$ & $15 \cdot 5$ & $21 \cdot 1$ \\
\hline
\end{tabular}

Das Endstadium wird also durch Zusatz von Salmiak nicht berïhrt, durch Lithiumchlorid merklich gemindert.

Je $1 \mathrm{~cm}^{3} 25 \%$ Malë̈nsâure vermischt mit $1 \mathrm{~cm}^{3}$ Fumarwasser und $4 \mathrm{~cm}^{3}$ 10 fach N. Salpetersünre. 1 blieb für sich, 2 mit überschüssigem Ammonium nitrat, 3 mit Kalisalpeter in Substanz 12 Tage stehen. 1 hatte dann reichlich, 2 nichts, 3 sehr wenig abgeschieden. 


\section{Versuche bei erhöhtem Druck.}

Da Schwefelsäure bei gewöhnlicher Temperatur nur bei solchen Concentrationen merklich wirkt, die äquimolecular Oxalsäure nicht liefern kann, wurden Versuche mit diesen zwei Säuren und mit Benzolsulfonsäure in zugeschmolzenen Röhren bei $130^{\circ}$, beziehungsweise $135^{\circ}$ durchgefuhrt.

$1 \mathrm{~cm}^{3} 25 \%$ Maleïnsüure vermischt mit $4 \mathrm{~cm}^{3}$ : Fumarwasser 1), 2fach N. Oxalsäure 2), 2 fach $\mathrm{N}$. Benzolsulfonsäure 3). 2 Stunden auf $100^{\circ}$ erhitzt, keine Veränderung, dann 21/2 Stunden auf 120 $0^{\circ}$, Abscheidung bloss in 2), $21 / 2$ Stunden auf $130^{\circ}$ : Abscheidung in allen Röhren. Titration der Fumarsäure nach 8 Tagen.

$\begin{array}{cccc}1 & 2 & 3 \\ \mathrm{~cm}^{3} 1 \frac{11}{10} \mathrm{NaOH} \ldots \ldots \ldots & 7 \cdot 0 & 10 \cdot 9 & 4 \cdot 0\end{array}$

In einem zweiten Versuch ist ausserdem eine Röhre mit Sehwefelsäure 4) eingeschaltet und $31 / 2$ Stunden auf $135^{\circ}$ erhitzt worden. Titration nach 11 Tagen.

\begin{tabular}{|c|c|c|c|}
\hline & 1 & 2 & 3 \\
\hline$c m^{3} 11 / 10 \mathrm{NaOH}$ & $6 \cdot 0$ & $9 \cdot 5$ & $4 \cdot 8$ \\
\hline
\end{tabular}

Übereinstimmend wirkte bloss die Oxalsätire beschleunigend. Benzolsulfonsäure und Schwefelsäure verzögern.

Vor der Titration sind den geöffneten Röhren Fliissigkeitsproben entnommen und deren elektrische Leitfähigkeit bestimmt worden. Hiebei habe ich mich der liebenswürdigen Unterstützung: des Herrn Svante Arrhenius zu erfrenen gehabt, dem ich für Rath und Hilfe auch an dieser Stelle freundlichst danke. Dieser Umstand enthebt mich auch einer Beschreibung der Versuchsanordnung und sei bloss Folgendes erwähnt:

Die Widerstandsbestimmungen gesehahen zuerst bei $22^{\circ}$, dann bei 58 , 96 und wieder bei $22^{\circ}$. Bei der Oxalsäure musste, um die in Lösung befindliche Kohlensäure zu entfernen, vorher auf $100^{\circ}$ erhitzt werden, um die Messungen zu ermöglichen.

Mit Hilfe von Interpolationen wurde aus den Zahlen von Kohlrauseh (Wiedemann, Elektricität) für 2 fach N. $\mathrm{H}_{2} \mathrm{SO}_{4}$ bei einer Temperatur von $21 \cdot 3^{\circ}$ die Leitfähigkeit von $3543.10-8$ S. E. berechnet. Mit dieser Zahl wurden die beobachteten Zahlen anf Leitungsvermögen in $\mathrm{S}$. E. bei den obenstehenden Temperaturen umgerechnet, wozn wieder Inter- und Extrapolationen nothwendig waren, und endlich auch für die (corr.) Temperatur von $134^{\circ}$, bei der die Umwandlung der Maleinsäure durch Erhitzen erfolgt ist. 
$22^{\circ} \quad 58^{\circ} \quad 96^{\circ} \quad$ Ber. f. 134

1) Maleỉnsäure allein. . . . . . . 231.10-8 $343.10-8 \quad 403.10^{-8} 463.10-8$

2) $n \quad$ mit Oxals.....491, 691,787 " 883 "

3) " $"$ Benzolsulfons. $3485 " 5520 " 7335 " 9150 "$

4) " "Schwefels....3568 " $4865 "$ "

Die für die Temperatur von $134^{\circ}$ aus den für 58 und $96^{\circ}$ gefundenen berechnete Zahl kann von der wahren Leitfähigkeit bei 134 (beziehungsweise 130) nicht erheblich abweichen.

In runder Zahl drückt sich das Verhältniss der Leitungsvermögen von Maleïnsäure allein : Oxalsäure : Schwefelsäure : $:$ Benzolsulfons $=1: 2: 14: 20$ aus, während die abgeschiedenen Mengen von Fumarsäure im Verhältniss $1: 1 \cdot 6: 0 \cdot 7: 0 \cdot 8$ stehen.

Dies mit den fruiber mitgetheilten Daten für die starken Mineralsäuren zusammengefasst, zeigt zweifellos, dass die Umwandlung der Maleïnsäure in Fumarsäure durch Säuren mit dem Leitungsvermögen in directem Zusammenhange nicht steht.

Es ist aber nicht unmöglich, dass bei grosser Verdünnung die Umwandlung vom Leitungsvermögen doch abhängig: ist. Hiefür spricht die Thatsache, dass, wie früher beschrieben ist, die grossen Unterschiede der Salz-, Salpeter- und Schwefelsäure nur bei grosser Concentration bestehen, bei abnehmender aber sich anuähernd ausgleichen. Dafür lässt sich auch der Verlauf einer anderen Versuchsreibe geltend machen, bei welcher leider in Folge eines Versehens die Bestimmung, welche allen Zweifel hätte Iösen können, unausführbar blieb.

Je $25 \mathrm{~cm}^{3}$ Normalmaleïnsäure wurden mit $25 \mathrm{~cm}^{3}$ von Normalsalz-, Salpeter-, Schwefel-, Oxals. und Benzolsulfons. in Fläschchen gefullt, die eine Spur fester Fumarsäure enthielten und mit Kautschukstöpsel verschlossen, in einen auf $25^{\circ}$ gehaltenen Thermostaten kamen. Alle Lösungen sind vorher mit Fumarsäure gesättigt nnd das Gleichgewicht durch Titration constatirt worden. Titrirt wurde mit sehr verdünntem Barytwasser. Die Differenzen betrugen nie mehr wie ein Zehntel Cubikcentimeter.

Der Titre der Gemische nabm so langsam ab, dass ich in meiner vorläufigen Mittheilung die Änderung nicht zu bebaupten

1 Die Leitfähigkeit nahm während des Versuches ab, was möglicherweise von einem Gase herrührt, das aber beim Herausnehmen des Gefüsses nicht wahrzuehmen war. 
wagte. Im Verlaufe von 10 Monaten ist sie aber deutlich genug. und unter Verbältnissen zu Tage getreten, die einen Irrthum ausschliessen dürften.

Gemisch gleicher Volume

Titre am

von N. Maleïnsäure mit Normal-

$12 / 5$

$27 / 7 \quad 11 / 10$

$25 / 2$

1. Salzsäure ...............21.72

$21 \cdot 27$

$21 \cdot 15$

$21 \cdot 00$

2. Salpetersäure

$22 \cdot 19$

$21 \cdot 71$

$21 \cdot 39$

$21 \cdot 2$

3. Schwefelsäure .............. 21:59

$21 \cdot 54 \quad 21 \cdot 49$

$21 \cdot 36$

4. Oxalsäure .................. 21 70

$21 \cdot 72$

$21 \cdot 67$

$21 \cdot 35$

๖. Maleînsäure

$21 \cdot 68$

$21 \cdot 78$

$21 \cdot 61$

Leider ist mir der auf sublimirte Oxalsäure gestellte Anfangstitre der Barytlösung verloren gegangen, es war daher nicht möglich zu constatiren, ob dieser in so langer Zeit keine Änderung erfahren hat. Doch selbst wenn man eine solche in Betracht ziehen wollte, bliebe es unerklärt, warnm der Titre verschiedener Säuren in Gefässen derselben Glassorte gegenüber der gleichen Barytlösung so ungleich abgenommen habe. Selbstverständlich kommt auch die Einwirkung der Säuren und des Wassers auf dus Glas in Betracht. Da aber nach Emmerling Schwefelsäure Glas am stärksten angreifen soll, so kann jene auch nicht erklären, warum Schwefelsäure den Titre geringer ändert als Salz- und Salpetersäure. ${ }^{1}$

Endlich glaube ich mich darin nicht zu täuschen, dass die schliesslich am Boden der Gefässe 1 und 2 befindliche feste Fumarsäure beträchtlicher war als die eingetragene und die einzelnen Kryställehen an Grösse zugenommen hatten.

Nimmt man eine katalytische Wirkung der Säuren auf die Maleïnsäure an, so steht das Mass dieser bei den einzelnen Säuren mit ihrem Leitungsvermögen in so guter Übereinstimmung, als unter den gegebenen Verhältnissen erwartet werden kann.

Ich halte es für möglich, dass die katalytische Wirkung darin besteht, dass entsprechend dem Leitungsvermögen Äpfelsäure gebildet wird und in Folge dieses Überganges geradeso wie bei

1 Die Gefässe waren zum Schlusse innen und aussen blank wie vor dem Versuche. Würde die Titreäncerung in $\alpha$ allein von der Auflösung des Glases herrïhren, müssten im Inneren $0.4 \mathrm{~g}$ Glas in Lösung gegangen sein, was eine nicht zu über'sehende Änderung der Oberfäche hätte herbeifïhren müssen. 
der Einwirkung von reinem Wasser bei höherer Temperatur ein Theil der Maleïnsäure in Fumarsäure übergeht.

\section{Organische Säuren.}

DieThatsache, dass Oxalsäure rascher umlagert als Schwefelsäure und Benzolsulfonsäure, konnte mit der Zersetzlichkeit der ersteren in Zusammenbang stehen. Besondere Versuche zeigten nämlich, dass Oxalsäure bei Wasserbadwärme ohne jede merkliche Wirkung ist, und dass anderseits, wenn bei höherer Tem. peratur Fumarsäure entsteht, immer die Zerfallsproducte der Oxalsäure, Kohlendioxyd und Ameisensäure nachweisbar sind. Darüber konnten Versuche mit Fettsäuren und Säuren der Oxalsäurereihe Aufschluss bringen.

Fettaäuren. In einer Versuchsreihe wurden je $4 \mathrm{~cm}^{3} 2$ fach normaler Lösungen von Ameisensäure 1), Essigsäure 2), Normalbuttersäure 3), Isobuttersäure 4), Oxyisobuttersäure 5) mit $1 \mathrm{~cm}^{3} 25 \%$ Maleïnsäurelösung $31 / 2$ Stunden auf $125^{\circ}$, in einer zweiten Reihe ebensolang auf $160^{\circ}$ erhitzt und jedesmal ein Rohr, das auf $1 \mathrm{~cm}^{3}$ Maleïnsëurelösung $4 \mathrm{~cm}^{3}$ Fumarwasser enthielt 6), zugefügt. Die Röhren öffneten sich ohne Druck, die Bestimmung der Fumarsäure geschah nach 5 , beziehlich 8 tägigem Stehen.

\begin{tabular}{|c|c|c|c|c|c|c|}
\hline & \multicolumn{6}{|c|}{ Verbrauch ron $1 / 10 \mathrm{NaOH}$} \\
\hline & 1 & 2 & 3 & 4 & $\overline{\mathrm{\jmath}}$ & $\ddot{6}$ \\
\hline Erhitzen ant $125^{\circ}$ & $11 \cdot \overline{0}$ & $7 \cdot 6$ & $0 \cdot 6$ & $6 \cdot 9$ & $14 \cdot 5$ & $8 \cdot 1$ \\
\hline$\Rightarrow \quad \Rightarrow 160^{\circ}$ & $20 \cdot 4$ & $14 \cdot 9$ & $9 \cdot 0$ & $13 \cdot 0$ & $20 \cdot 2$ & $11 \cdot 7$ \\
\hline
\end{tabular}

Die Säuren, die schwächer nmwandeln, ja, die Reaction, die Maleïnsüure mit Wasser allein gibt, verzögern, sowie jene, die sie beschleunigen, sind in beiden Reihen dieselben, so dass ein Ziffall ausgeschlossen werden kann.

Mehrbasische Säuren. Da isomere mehrbasische Säuren häufig verschieden leicht Kohlendioxyd abspalten, konnten Versuche mit solchen Aufschluss bringen, ob die Unwandlung der Maleänsäure durch Oxalsäure mit der Kohlensäureabspaltuug in Zusammenhang steht.

Je $1 \mathrm{~cm}^{3} 25 \%$ Maleünsäure mit $4 \mathrm{~cm}^{3}$ 1) Fumarsäure 2) 2 fach N. Malonsäure, 3) 2 fach N. Isobernsteinsäure $31 / 2$ Stunden auf $135^{\circ}$. Nach 42 Stunden wurde die Fumarsäure bestimmt.

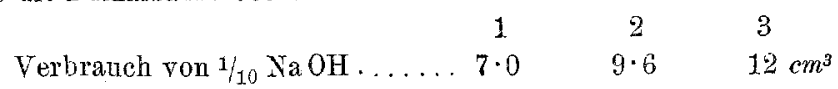

Malonsüure und Isobernsteinsüure beschleunigen die Umsetzung also geradeso wie Oxalsäure.

In den Röhren ‘ und 3 war starker Druck. Die entweichende Kohlensiuue vermittelst einer Luftpumpe in ein Natronkalkrohr gefübrt, zeigten 
nahezu genau dasselbe Gewicht wie jene, die aus Röhren ausströmte, in welcher die gleich concentrirten Säuren ohne Maleïnsäurezusatz in gleicher Art erhitzt worden waren.

Die isomeren Glutarsäuren sind in Wasser so weit löslich, dass doppelt Normullösungen bereitet werden können. Versuche mit thnen verliefen folgendermassen:

A. Je $1 \mathrm{~cm}^{3} 25 \%$ Maleïnsäure vermischt mit $4 \mathrm{~cm}^{3} 2$ fach Normal 1), Ghutar-2) Brenzwein- 3), Dimethyl- 4), Äthylmalonsäure 5), Fumarwasser $31 / 2$ Stunden anf $135^{\circ}$ erhitzt.

$B$. Ansserdem wurden je $4 \mathrm{~cm}^{3}$ der Säuren 1, 2, 3, 4 mit $1 \mathrm{~cm}^{3}$ Fumarwasser in demselben Trockensehrank mit erbitzt.

$$
\begin{aligned}
& \begin{array}{llll}
1 & 2 & 3 & 4
\end{array} \\
& \text { Es entwich } \mathrm{CO}_{2} \text { aus: } A \ldots \ldots \cdot 0 \cdot 0035 \quad 0.003 \quad 0 \cdot 1035 \quad 0 \cdot 1180 \mathrm{~g} \\
& \begin{array}{llllll}
B \ldots & 0 \cdot 0000 & 0 \cdot 000 & 0 \cdot 1730 & 0 \cdot 1711
\end{array}
\end{aligned}
$$

Nach 8 Tagen wurde die Fumarsïure bestimmt:

$\begin{array}{cccccc}1 & 2 & 3 & 4 & 5 \\ \text { Verbrauch vou } 1 / 10 \text { Na OH } \ldots . . .10 \cdot 2 & 3 \cdot 0 & 2 \cdot 6 & 6 \cdot 4 & 9 \cdot 55\end{array}$

Für den vollständigen Zerfall der Glutarsäuren unter Abgabe von $1 \mathrm{Mol} . \mathrm{CO}_{2}$ berechnet sich $0 \cdot 176, g \mathrm{CO}_{2}$. Es scheint also bei Gegenwart von Maleïnsäure die Zersetzung schwieriger zu verlaufen als bei deren Abwesenheit.

Die abgeschiedenen Mengen von Fumarsäure bediurfen für 3 and 4 einer Correctur, da das Lösungsmittel nach dem Erhitzen eine andere Zusammensetzung bat als vorher. Ich habe dieselbe derart zu ermitteln gesucht, dass ich feststellte, wie viel Fumarsäure die Flüssigkeiten der Röhren 3 und 4 der Reihe $B$ nach dem Erhitzen anfzunehmen vermögen. Mit Hinzurechnung dieser Mengen stellt sich in den Versuehen $A 1$ bis 5 die abgeschiedene Fumarsäure, bezichungsweise die verbranchte Menge $1 / 10 \mathrm{NaOH} z \mathrm{a}$

$\begin{array}{ccccc}1 & 2 & 3 & 4 & 5 \\ 10 \cdot 2 & 8 \cdot 0 & 6 \cdot 3 & 7 \cdot 6 & 9 \cdot 55\end{array}$

Die Wirkung verschiedener mehrbasischer organischer Säuren ist demnach ziemlich gleich und merklich nicht sehr verschieden von der des Wassers.

Beim Erwärmen von Maleïnsäurelösungen mit Phloroglucinearbonsäure und mit einem äquivalenten Gemisch von dLoxyweinsaurem Natrium und verdüunter Schwefelsäure ist die Bildung von Fumarsäure nicht zu beobachten gewesen, was neuerdings bestätigt, dass die Abspaltung von $\mathrm{CO}_{2}$ ohne Wirkung ist. Das dioxyweinsaure Natritm verdanke ich der Güte des Herrn Directors C. Glaser, dem ich auch an dieser Stelle bestens danke. 
Organische Säuren üben also durchwegs geringen Einfluss, und die kleinen Differenzen ihrer Wirkung lassen sich vorläufig nicht erklären.

Die Umwandlung der Maleïnsäure durch Säuren könnte vielleicht auch derart gedeutet werden, dass diese voribergehend Äpfelsäure hilden, die unter Wasserabspaltung Fumarsäure liefert. Obzwar schon die bisher beschriebenen Beobachtungen gegen eine solche Auslegung sprechen, habe ich doch noch einige Versuche ausgeführt, die zeigten, dass eine $25 \%$ Äpfelsäurelösung in den verschiedensten Verhältnissen mit Salz-, Salpeter- und Schwefelsäure vermischt, bei gewöhnlicher Temperatur Fumarsäıre auch nach vielwöehentlichem Stehen nicht abscheidet, während ganz analog gemischte Maleïnsäurelösungen reichlich abgeschieden haben.

Stellt man die Resultate, die dieser Abschnitt geliefert hat, zusammen, so ergibt sich erstlich, dass nur jene Säuren die Vale rnsäure leicht in Fumarsäure verwandeln, die auf jene leicht chemisch einzuwirken vermögen.

Bei den Halogenwasserstoffsänren lässt sich unzweideutig verfolgen, dass Umwandlung and Entstehung eines Additionsproductes gleichzeitig sind, dass also beide Vorgänge gesetzmässig in Verbindung stehen, nachdem ganz dasselbe für die Einwirkung von Wasser, sowie fiir die Einwirkung von Brom gilt und gewiss ebenso für die Einwirkung von Chlor richtig sein dürfte, nachdem ferner für einige dieser Reactionen festgestellt and für andere sehr wahrscheiniich ist, dass die entstehendeu Additionsproducte in Folge ihrer relativen Beständigk eit als Zwischenformen nicht in Frage kommen, muss auf einen katalytischen Zusammenhang der nebeneinander laufenden Processe geschlossen werden.

Die Gründe, die dafür sprechen, dass die Addition der ursächliche und die Umlagerung der katalytisch angeregte Process ist, brauchen wohl nicht besonders erörtert zu werden. Einige Schwierigkeiten macht es, die freilich nur geringe Verschiedevheit in der Wirkung organischer Sätren, die Thatsache, dass manche Säuren die Wirlsung reinen Wassers herabmindern, endlich zu erklären, dass Salpetersätre, von der ein Additionsproduct weder erwartet, noch nachgewiesen werden konnte, die Umwandlung so erheblich bewirkt. 
Ich kann es also nur als Vermuthung hinstellen, dass die Salpetersäure dadurch katalytisch wirkt, dass sie einen Theil der Maleïnsäure oxydirt; die Beobachtungen der folgenden Abschnitte dürften diese Vermuthung unterstiitzen.

\section{Einwirkung von Schwefelwasserstoff auf Salze der Maleïn- säure.}

Die Salze mit Schwermetallen setzen sich mit Schwefelwasserstoff sehr leicht $\mathrm{nm}$, wenn sie fein gepulvert sind und beim Durchleiten des Gases umgeschüttelt wird. Das Filtrat vom Yetallsulfid eingedampft und mit fumarsäurehältigem Wasser auf ein solehes Volum (in der Regel 5 oder $10 \mathrm{~cm}^{3}$ ) gebracht, dass die Maleïnsäure leicht in Lösung geben kann, scheidet ausnahmslos Fumarsäure ab. Obzwar die physikalischen Eigenschaften der schwer löslichen Säure und ihr Verbalten beim Erhitzen über ibre Natur keinen $Z$ weifel liessen, habe ich doch in zwei Fällen die Elementaranalyse ausgeführ, welche scharf stimmende Werthe ergab. Die relative Menge von Fumarsäure ist einmal von der Natur des Metalls, welches in das Sulfid übergeht, ausserdem aber noch von einer Reihe anderer Umstände abhängig, welche genau festzustellen nicht gelungen ist. Dem naheliegenden Einwurf, die verwendeten Salze hätten von vornherein fumarsaures Salz entbalten, können die Thatsachen begegnen, dass erstlich zu ihrer Darstellung möglichst reine Maleïnsäure ${ }^{1}$ in Anwendung gekommen ist, dass zweitens die Salze in einer mit Fumarsäure gesättigten Lösung, die freie Mineralsäure enthält, vollständig auflöslich waren, dass es sich in den meisten Fällen nicht um eine spurenweise, sondern oft sehr bedentende Umwandlung handelt und letztere überdies bei ein- und demselben Präparat

1 Aus der käuflichen Maleïnsäure, die stets, oft viel Fumarsäure enthält, kann durch Umkrystallisiren ein ganz reines Präparat nicht erhalten werden. Die Maleïnsäure war uberall dort, wo es sich um die Abwesenheit jeder Spur von Fumarsäure gehandelt hat, derart gereinigt, dass Malë̈nsäureanhydrid in einer unzureichenden Menge Chloroform in der Kälte gelöst, die von dem Ungelösten, das Fumarsäure enthält, abgegossene Lösung theilweise abdestillirt, das Auskrystallisirte nochmals in gleicher Weise behandelt, endlich in wenig lauwarmem Wasser in die Säule übergeführt wurde. Die so erhaltenen grossen Krystalle lösen sich in Fumarwasser absolut klar auf. 
sehr verschieden gefunden wurde, wenn noch secundäre Einflüsse auftraten.

\section{Maleinsaures Kupfer.}

4 Antheile von je $1 \mathrm{~g}$ maleïnsaurem Kupfer (Nummern 1 bis 4, 2 und 4 mit je $2 g$ Kupfercarbonat vermischt) wurden mit $25 \mathrm{~cm}^{3}$ Wasser übergossen, 1 und 2 in der Kälte, 3 und 4 kochend mit Schwefelwasserstoff zerlegt, Das Einleiten wurde fortgesetzt, bis die Flüssigkeiten auch nach einigem Stehen nach Schwofelwasserstoff dentlich rochen. Die Filtrate (von 1, 2 nnd 4 dunkelgrïn gefärbt) wurden eingedampft, bis das colloidale Schwefelkupfer filtrirbar war, dann zur Trockene und mit Wasser auf $5 \mathrm{~cm}^{3}$ gebracht.

$\begin{array}{rcccc}\text { Zur Titration der Fumarsäure wurden } & 2 & 3 & 4 \\ \text { gebraucht } \mathrm{cm}^{3} 1 / 10 \text { Na OH ........ } & 35 \cdot 5 & 37 \cdot 7 & 19 \cdot 1 & 60 \cdot 3 \\ \text { Fumarsäure gebildet in Procenten ... } 35 \cdot 1 & 37 \cdot 7 & 20 \cdot 7 & 57 \cdot 2\end{array}$

Maleïnsäure war in allen vier Operationen unverändert geblieben; in Versuch 3 war gar kein, in 1 und 2 wenig, in 4 viel colloidales Schwefelkupfer in Lösung gegangen.

Eine ungewogene Menge Kupfersalz wurde im kochenden Wasserbad nicht länger mit Schwefelwasserstoff behandelt, bis das blaue Pulver vollständig sohwarz geworden war. Lösung wie oben auf $5 \mathrm{~cm}^{3}$ gebracht, nach zwei Tagen wurde der Titre der Lauge über der abgeschiedenen Fumarsäure und diese selbst bestimmt. Die Lösung war so gut wie frei von Schwefelsäure.

$1 \mathrm{~cm}^{3}$ Lösung brancht $13 \cdot 00 \mathrm{~cm}^{3}$, die Fumarsänre $24 \cdot 5 \mathrm{~cm}^{3} 1 / 10 \mathrm{NaOH}$. Umwandlung $27 \cdot 37$.

a) $1 g$ Maleïnsäure in $25 g$ Wasser gelöst, mit $1 g$ Kupfercarbonat bis zur Beendigung der Kohlensäureentwickelung digerirt, im kochenden Wasserbad mit $\mathrm{SH}_{2} 11 / 2$ Stunden behandelt, letzterer im $\mathrm{CO}_{2}$-Strome verdrängt, im Vacuum eingeengt, filtrirt, auf $10 \mathrm{~cm}^{3}$ gebracht.

b) $1 \mathrm{~g}$ Maleïusäure mit $10 \mathrm{~g}$ Kupfercarbonat, sonst wie oben behandelt, während dem Einleiten noch $30 \mathrm{~g}$ Wasser zu, um den dicken Brei im Kolhen etwas dünner zu machen. Beide Proben wurden mit Wasser auf $10 \mathrm{~cm}^{3}$ gebracht.

In $a$ ) und $b$ ) Maleïnsäure noch unverändert, die abgeschiedene Fumarsäure nahezu gleich viel. Durch einen Unfall wurde die directe Bestimmung. vereitelt, desshalb eine indirecte durch Ermittelung des Titres der Lösung uiber der ausgeschiedenen Fumarsäure vorgenommen, die für b) etwas zu niedrige Werthe ergeben musste, da die Lösung b) Schwefelsäure enthielt.

12

Titre der Lösung in $\mathrm{cm}^{3} 1 / 10 \mathrm{NaOH} \ldots \ldots .6 .99 \quad 8.84$

Fumarsäure in Procenten..........64.1 $53 \cdot 6$ 
Maleïnsaures Kupfer scheidet demnach unter allen Umständen neben Malë̈nsäure Fumarsäure ab, umsomehr, je länger Schwefelwasserstoff eingeleitet wurde, und scheint die Bildung von colloidalem Schwefelkupfer die Umwandlung in Fumarsäure zu begiunstigen. Die Umsetznng von beigemischtem Carbonat in Schwefelkupfer hat dagegen keinen Einfluss.

\section{Maleinsaures Blei.}

a) Ungewogene Menge rasch zerlegt mit Fumarwasser auf $5 \mathrm{~cm}^{3}$. Titre der Lösung $32 \cdot 91 / 10 \mathrm{Na} \mathrm{OH}$, Fumarsäure brancht $39 \cdot 0 \mathrm{~cm}^{3}$. Umwandlung $19 \cdot 6 \%$.

b) $1.5 \mathrm{~g}\left(=0 \cdot 464\right.$ Säure) mit $9 g$ Bleiweiss (aus Bleiessig mit $\mathrm{CO}_{2}$ anisgefällt), 15 Minuten im $\mathrm{SH}_{2}$-Strom (1), dann $1.5 g$ ohne Bleiweisszusatz (2) ebensolang behandelt. Beide mit Fumarwasser auf $10 \mathrm{~cm}^{3}$.

$\begin{array}{lcc} & 1 & \\ \text { Fumarsäure brauchte } \mathrm{cm}^{3} 1 / 10 \mathrm{Na} \mathrm{OH} \ldots \ldots & 25 \cdot 4 & 25 \cdot 2 \\ \text { Umwandlung in Procenten } \ldots \ldots \ldots \ldots \ldots & 31 \cdot 7 & 31 \cdot 5\end{array}$

Das Bleisalz verhält sich dem Kupfersalz ganz gleich. Die vermehrte Bildung von Metallsulfid aus dem Carbonat ist auch bier ohne Einfluss.

\section{Silbersalz.}

a) $1 g$ Salz $(=0 \cdot 353$ Sänre), dargestellt durch Füllen des Ammonsalzes, zerlegt und mit Fumarwasser auf $5 \mathrm{~cm}^{3}$ gebracht.

b) $2.0803 g$ Salz ( $=0.7357 g$ Säure), dargestellt aus reinem Baryumsalz, zerlegt und mit Fumarwasser auf $10 \mathrm{~cm}^{3}$ gebracht.

\begin{tabular}{|c|c|c|}
\hline & $a$ & $b$ \\
\hline Fumarsäure braucht $\mathrm{cm}^{3} 1 / 10 \mathrm{NaOH}$ & $4 \cdot 0$ & $16 \cdot 9$ \\
\hline Umwandlung $\ldots \ldots \ldots \ldots \ldots$ & $6 \cdot 6 \%$ & $13 \cdot 2 \%$ \\
\hline
\end{tabular}

IV. Zerlegung verschiedener Salze unter möglichst gleichen Bedingungen.

Da das Silbersalz auffallend weniger Fumarsäure abschied als die Salze des Kupfers und des Bleies, erwies sich nothwendig. festzustellen, ob auch sonst die Natur des Metalls von Einfluss sei. Um möglichst gleiche Bedingungen zu erreichen, sind äquivalente Mengen der Salze (auf $1.5 \mathrm{~g}$ Kupfersalz berechnet) mit je $25 \mathrm{~cm}^{3}$ Wasser in demselben Fractionskolben nicht länger im Schwefelwasserstoffstrom bei Wasserbadwärme behandelt worden, 
bis kein unzersetztes Salz wahrnehmbar war; es danerte dies in allen Fällen etwa 7 Minuten. Hierauf jst vermittelst eines Dreiweghahnes der Schwefelwasserstoffstrom durch Kohlendioxyd ersetzt, dies etwa 10 Minuten durchgeleitet, die Lösung dann vor der Pumpe filtrirt, gleichmässig mit luftfreiem Wasser verdrängt, eingedampft, der Rückstand mit Fumarwasser auf $5 \mathrm{~cm}^{3}$ gebracht, die Fumarsäure nach zwei Tagen filtrirt und bestimmt worden.

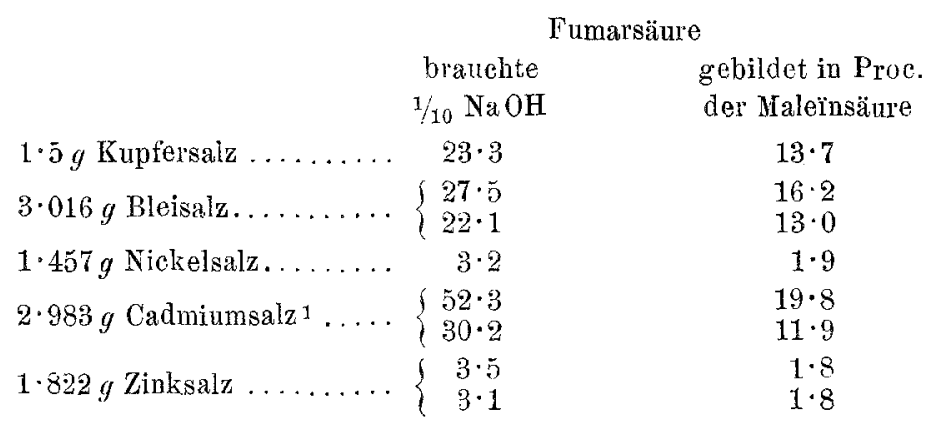

Obzwar trotz möglichst gleichmässigem Arbeiten bei ein und demselben Metallsalz nicht unerhebliche Abweichungen vorliegen, die einen genauen Vergleich erschweren, so sind doch die Differenzen zwischen den Salzen verschiedener Metalle so gross, dass Versuchsfehler nicht angenommen werden können und desshalb die Natur des Metalls auf den Grad der Umwandlung zweifellos bestimmenden Einfluss hat.

Es liegt nahe, die relativen Mengen Fumarsäure, die bei den verschiedenen Reactionen entstehen, mit den Wärmetönungen der letzteren in Vergleich zu setzen. Abgesehen davon, dass die ermittelten Zahlen zweifellos nur annähernde Richtigkeit haben werden, fehlen aber auch eine Reihe thermochemischer Werthe, und desshalb ist der Vergleich nicht möglich.

Bei den beschriebenen Versuchen ist die von der Fumarsänre getrennte Lösung, die Maleïnsäure enthielt, jedesmal auf eine Ver-

1 Das durch Eintragen von Cadmiumcarbonat in eine warme Lösung von Malë̈nsäure, Filtriren vom Ungelösten und Eindampfen bis zur Krystallisation erhaltene Salz ist nicht das Neutrale, sondern ein schwer definirbares Gemisch von viel saurem und etwas neutralem Salz. Bei der Berechnung in obiger Tabelle ist die aus den Analysenwerthen sich ergebende Menge an Säure in Rechnung gezogen. 
unreinigung durch Salzsäure und Schwefelsäure geprüft worden. Mit Ausnabme zweier Einzelnversuche, bei denen die Abweichung schon bemerkt ist, waren die Lösungen von den zwei Säuren so gut wie frei. Im äussersten Fall trat beim Nachweis eine leichte Opalescenz ein.

Die Überführung der Maleïnsäure kann also durch Schwefelsäure, die durch Oxydation des Schwefelwasserstoffes entstehen kann, oder durch aus dem Entwickelungsapparat mitibergerissene Salzsäure nicht bewirkt sein. Ausserdem babe ich schon früher nachgewiesen, dass verdünnte Schwefelsäure auf Maleïnsäure beim Erwärmen so gut wie ohne Wirkung ist.

Man kann die Entstehung der Fumarsäure auch nicht als eine allgemein giltige Wirkung ansehen, die eintritt, wenn die Säure durch ein beliebiges Mittel frei wird, da das Silbersalz wohl Fumarsäure liefert, wenn es mit Schwefelwasserstoff, nicht aber, wenu es mit Salzsäure zerlegt wird.

$2 \cdot 3412 g$ desselben Silbersalzes, das, mit Schwefelwasserstoff zerlegt, $13 \cdot 3 \%$ der Säure als Fumarsälire abschied, sind mit Wasser übergossen und bei gewöhnlicher Temperatur mit 1/10 N. Salzsäure genau zerlegt worden. Die eingedampfte Säure mit Fumarwasser auf $10 \mathrm{~cm}^{3}$ gebracht, schied nicht die Spur einer unlöslichen Verbindung ab, eine Umwandlung war also nicht eingetreten.

Anderseits liefert das Barytsalz der Maleïnsäure, mit Schwefelsåure zerlegt, Fumarsäure.

$2 \cdot 1565 g$ in Wasser gelöst und mit der aus dem Barytgehalt (wie er durch Analyse festgestellt wurde) berechneten Menge $1 / 10 \mathrm{~N}$. $\mathrm{H}_{2} \mathrm{SO}_{4}$ vermischt und mit Fumarwasser auf $10 \mathrm{~cm}^{3}$. Die abgeschiedene Fumarsäure verbrauchte $0.8 \mathrm{~cm}^{3} 1 / 10 \mathrm{NaOH}$, entsprechend einer Umlagerang von $0 \cdot 5 \%$.

Bei manchen der beschriebenen Versuche ist eine Vermehrung: der Fumarsäure dann eingetreten, wenn länger Schwefelwasserstoff eingeleitet wurde, als zur Zerlegung unbedingt nothwendig war. Um festzustellen, ob und in welchem Grade Schwefelwasserstoff Einfluss hat, dienten folgende Versuche:

1. $1 \mathrm{~g}$ Maleïsäure in $100 \mathrm{~g}$ Wasser $11 / 2$ Studen im Schwefelwasserstoffstrom bei Wasserbadwärme, dann 1 Stunde mit $\mathrm{CO}_{2}$ behandelt, eingedampft und mit Fumarwasser auf $5 \mathrm{~cm}^{3}$ gebracht.

2. $2 \mathrm{~g}$ Maleïnsäure in $10 \mathrm{~cm}^{3} \mathrm{H}_{2} \mathrm{O}$ 1/2 Stunde kalt, $1 / 2$ Stunde heiss, dann wieder $1 / 2$ Stunde kalt mit Schwefelwasserstoff behandelt, eingedampft und auf $10 \mathrm{~cm}^{2}$ gebracht. 
3. 1 g Maleinsäure genau so behandelt wie die unter IV. beschriebenen äquivalenten Hengen. Salze, mit ebensoviel Wasser verdünnt, als sonst zum Waschen verwendet wurde, dann auf $4 \mathrm{~cm}^{3}$ gebracht.

4. 1 g Malë̈nsäure $1 / 2$ Stunde in der Kälte mit $\mathrm{SH}_{2}$ eingedampft, auf' $5 \mathrm{~cm}^{3}$ gebracht.

$\begin{array}{llcccc} & 1 & 2 & 3 & 4 \\ \text { Fumarsäure braucht } c m^{3} 1 / 10 \mathrm{Na} O \mathrm{H} \ldots \ldots & 6 \cdot 4 & 5 \cdot 5 & 0 \cdot 3 & 0 \\ \text { Umwandlung in Procenten. } \ldots \ldots \ldots \ldots & 3 \cdot 71 & 1 \cdot 59 & 0 \cdot 17 & 0\end{array}$

Bei den unter IV auf S. 135 beobachteten Bedingungen, unter welchen die Salze zerlegt wurden, ist Schwefelwasserstoff also so gut wie ohne Einfluss, ebenso in der Kälte, bei längerer Einwirkung in der Wärme bewirkt er aber deutlich Umwandlung.

Um über diese einigermassen Vorstellung zu erhalten, habe ich gleichzeitig mit dem in 2 . beschriebenen Versuch unter ganz denselben Umständen den Schwefelwasserstoffstrom in $10 \mathrm{~cm}^{3}$ reines Wasser und zwej andere Kölbchen treten lassen, von denen jedes eine Lösung von $2 g$ Maleïnsäure enthielt.

Eines der letzteren wurde durch eine halbe Stunde mit dem Kollensäureapparat verbunden, der Inhalt dann in eine Schale gespilt und uber Ätzkali ins Vactum gestellt. Über Nacht war jede Schwefelwasserstoffreaction verschwunden. Die Lösung mit iibersehiissigem Kaliumbicarbonat und Salpeter vermischt, eingedampft und schliesslich im Silberticgel verascht, gab eine kauma wahrnehmbare Schwefelsäurereaction.

Ein zweites Kölbchen mit Malë̈nsäurelösung und das mit Wasser babe ich gleichmässig mit Wasser vermischt, den Inhalt in ïberschüssiges Bromwasser gebracht und die durch Oxydation des Schwefelwasserstoffes entstandene Schwefelsäure bestimmt.

Reines Wasser gab .......... $0.2737 \mathrm{~g} \mathrm{Ba} \mathrm{SO}_{4}$,

die Maleïnsäurelösung ......... $0 \cdot 3868 \mathrm{~g} \mathrm{Ba} \mathrm{SO}_{4}$.

Die Malë̈nsäurelösung absorbirt also zwar mehr von dem Gase als Wasser, die eventuell entstehende Verbindung ist aber so locker, dass sie schon beim Stehen im Vacuum zerfällt.

Bei anbaltendem Erhitzen tritt aber bestimmt eine energischere Einwirkung ein. Je $2 g$ Maleïnsäure wurden einmal in Alkohol, das anderemal in Wasser gelöst, im Einschmelzrohr bei $-20^{\circ}$ mit Sehwefelwasserstoff gesättigt, dann 2 Stunden auf $100^{\circ}$ erhitzt, nach dem Öffnen abermals unter Kühlung mit Eis und Kochsalz mit dem Gase gesättigt und wieder erhitzt. 
Der Inhalt der Robren ist im Vacuum theilweise abdestillirt, dann am Wasserbad auf ein kleines Volum gebracht worden.

Der Rest der alkoholischen Lösung schied zuerst ein wenig: eines schwer Iöslichen Pulvers, anscheinend Fumarsäure $a b$ und binterliess dann eine sehr leicht lösliche Säure, auf welche die Eigenschaften der Thioäpfelsäure von Carius passen. Aus der wässerigen Flïssigkeit schossen dagegen ziemlich schwer lösliche Prismen einer schwefelhältigen Säure an, die keinesfalls die erwähnte Thiosäure sein kann. Über die Natur dieser Producte müssen weitere Untersuchungen Aufschluss bringen.

$O b$ die Bildung eines dieser Körper damit im Zusammenbange steht, dass bei länger fortgesetztem Einleiten in Maleînsäure mehr Fumarsäure entsteht, ist desshalb auch nicht zu entscheiden.

\section{Gleichzeitige Einwirkung von Schwefelwasserstoff und Schwefeldioxyd.}

Schwefelwasserstoff ist auf Maleïnsäurelösung bei mittlerer Temperatur von geringer Einwirkung. Dasselbe gilt, wie schon erwähnt, aber noch nicht näher beschrieben worden ist, vom Schwefeldioxyd.

$5 \mathrm{~cm}^{3} 25 \%$ Lösung $1 / 2$ Stunde in der Kälte, dann $1 \frac{1}{2}$ Strunden bei Wasserbadtemperatur mit $\mathrm{SO}_{2}$ behandelt, dann im Vacuum theilweise abdestillirt, eingedampft und mit Fumarwasser auf $5 \mathrm{~cm}^{3}$ gebracht. Fumarsäure braucht $2 \cdot 8 \mathrm{~cm}^{3} 1 / 10 \mathrm{NaOH}$. Umwandlung $1 \cdot 6 \%$.

$5 \mathrm{~cm}^{3}$ wie oben $1 / 2$ Stunde in der Kälte behandelt, dann 14 Tage stehen gelassen, eingedampft, wie oben behandelt. Fumarsäure braucht $0.95 \mathrm{~cm}^{3}$ $1 / 10 \mathrm{Na}$. OFC, Umwandlung $0.5 \%$.

Wird aber Maleïnsäurelösung mit Schwefeldioxyd und mit Schwefelwasserstoff gesättigt und erwärmt, so wird reichlich Fumarsäure gebildet.

a) $1 \mathrm{~g}$ Săure in $100 \mathrm{~cm}^{3}$ wässeriger schwefliger Säure gelöst, mit $\mathrm{SH}_{2}$ behandelt, bis dessen Geruch vorwaltet, eingedampft, bis der Schwefel filtrirbar ist, fast zur Trockene eingedampft und mit Fumarwasser auf $10 \mathrm{~cm}^{3}$ gebracht.

Fumarsäure brauchte $67 \cdot 6 \mathrm{~cm}^{3} 1 / 10 \mathrm{NaOH}$. Umwandlung $40 \cdot 40 \%$.

b) $2 \mathrm{~g}$ Maleïnsäure in $10 \mathrm{~cm}^{3}$ Wasser mit $\mathrm{SO}_{2}$, dann mit $\mathrm{SH}_{2}$ gesättigt, dann wie oben behandelt.

Fumarsäure braucht $65 \cdot 1 \mathrm{~cm}^{3}$. Umwandlung $18 \cdot 8 \%$. 
c) $10 \mathrm{~cm}^{3}$ Wasser mit $\mathrm{SO}_{2}$, dann mit $\mathrm{SH}_{2}$ gesättigt, oiugedampft, filtrirt, dann erst mit $2 g$ Maleïnsäure vermischt, dreimal unter Erneuerung des Wassers eingedampft, dann auf $10 \mathrm{~cm}^{3}$ gebracht.

Fumarsäure brancht $19 \cdot 2 \mathrm{~cm}^{3}$. Umwandlung $5 \cdot 5 \%$.

Da Schwefeldioxyd sowohl wie Schwefelwasserstoff unter analogen Verhältnissen keine oder doch nur sehr geringe Wirkung hab :n, diese aber in hohem Grade eintritt, wenn beide gleichzeitig: eing eleitet werden, so muss die Umwandlung der Maleïnsäure in dem chemischen Zusammenwirken beider Gase ihren G:und haben.

In $10 \mathrm{~cm}^{3}$ Wasser wurden, das eine Volum für sich, das andere rach dem Eintragen von $2 g$ Maleünsäure mit $\mathrm{SO}_{2}$ unter Kühlen mit Wasser gesättigt. Der Inhalt beider, pachdem sie gut versehlossen über Nacht gestanden waren, in Bromwasser eingetragen nnd mit $\mathrm{BaCl}_{2}$ gefällt.

Reines Wasser gab ..........3.7068 g $\mathrm{BaSO}_{4}$,

Malen̈nsäurelösung gab .......4.2555 $\mathrm{g} \mathrm{BaSO}_{4}$.

Maleïnsäure absorbirt also in wässeriger Lösung ebenso mehr $\mathrm{SO}_{2}$ wie reines Wasser, als $\mathrm{SH}_{2}$.

Mas man nun aber annehmen, dass das Plus an $\mathrm{SO}_{2}$ oder $\mathrm{SH}_{2}$ mit der Maleinsäure eine lockere Verbindung eingeht und diese dann mit dem zweiten Gase in einer ganz unerklärten Weise reagire und bei dem einen oder dem anderen Verlaufe Fumarsäure entstehen soll; so lassen sich die guantitativ ermittelten Verhältnisse damit doch in keiner Weise erklären.

Aus den mitgetheilten Daten berechnen sich auf 1 Mol. Maleïnsüure d.s. 116 Theile $8 \cdot 7$ Theile $\mathrm{SO}_{2}$ und aus den auf $\mathrm{S} .138$ veröffentlichten auf 116 Maleïnsäure 0.95 Theile $\mathrm{SH}_{2}$, die chemisch gebunden sein könnten.

Aus diesen Zahlen ergibt sich, dass wenn die Aetion von $\mathrm{SH}_{2}$ auf ein lockeres Additionsproduct zwischen Maleïnsäure und $\mathrm{SO}_{2}$ Fumarsäure liefern sollte, von letzterer unter den eingehaltenen Bedingungen nur $13 \%$ der Maleïnsäure entstehen könnte, im umgekehrten Falle bloss $2 \%$, während thatsächlich bei gleichzeitiger Einwirkung beider Gase 18\% gebildet wurden. Man muss also auch hier ausschliessen, dass intermediäre Additionsproducte bei der Umwandlung eine Rolle spielen und wird die Ursache dieser geradeso wie in anderen Fällen in der Energieänderung zu suchen haben, die bei der Einwirkung von $\mathrm{SO}_{2}$ und $\mathrm{SH}_{2}$ in wässeriger Lösung vor sich geht. 
Diese Ansicht wird durch den Umstand untersititzt, dass wie ein Vergleich zwischen den Versuchen $a$ ), b) und $c$ ), S. 139 und 140, lehrt, die Umwandlung umso bedeutender ist, je mehr der beiden Gase in Wirkung treten, und dass sie zurücktritt, wenn wie in c) die chemischen Processe schon grösstentheils abgelaufen waren, bevor die Maleïnsäure zukam.

Im Rahmen einer Untersuchung alle jene Reactionen anzugehen, von denen nach den gemachten Erfahrungen ein Einfluss auf die Umlagerung der Maleïnsäure zu erwarten steht, ist natürlich nnausführbar.

Ich beschränke mich, einige chemische Umsetzungen anzufuihren, denen ein Einfluss abgeht.

So verliefen resultatslos Versuche, die Umwandlung: der Maleïnsäure in Fumarsäure durch Erhitzen ibrer wässerigen Lösung mit Acetessigester, Essigester, dann Aldehyd und Paraaldehyd unter Zufügung einer Spur von Schwefelsäure $\left(1 / 10 \mathrm{~cm}^{3}\right.$ $1 / 10 \mathrm{~N}$ ) zu bewirken.

Dasselbe gilt von der Zerlegung des Essigsäureanhydrids durch Maleinnsäurelösung bei gewöhnlicher Temperatur oder bei $100^{\circ}$. Die quantitative Verfolgung zeigte, dass hiebei nicht die Spur Fumarsäure entsteht.

Dagegen ist ein Einfluss mancher Oxydationsprocesse wahrzunehmen. Mit Sicherheit gelang es jenen für die Oxydation des $\mathrm{SO}_{2}$ durch Salpetersäure nachzuweisen. Eine $25 \%$ Lösungig mit $1 / 5$ des Volums gew. $\mathrm{NO}_{3} \mathrm{H}$ vermischt und dann mit $\mathrm{SO}_{2}$ in der Kälte gesättigt, schied im Verlauf einiger Tage reichlich Fımarsäure $a b$, viel mebr als Salpetersäure derselben Concentration in derselben Zeit bei $A b w e s e n h e i t$ ron Schwefeldioxyd hätte bilden können. Ebenso war manchmal wahrzunehmen, dass Gemische von Maleïnsäure und Salpetersäure mehr Fumarsäure beim Er. wärmen abschieden, wenn ihnen Alkohol oder Aldebyd zugefitgt wurde, als ohne einen solchen Zusaty (siebe auch S. 124 über die Umwandlung durch Salpetersäure allein).

\section{Schlussbemerkungen.}

Die möglichst genaue Verfolgung der Umsiände, unter welchen Maleïnsäure beim Erhitzen mit Wasser, durch die Einwirkung von Jodwasserstoff, und Salzsäure in Fumarsäure übergeht, in Ver- 
bindung mit schon bekannten Verhältnissen, wie sie für die Wirkung von Bromwasserstoff und freiem Brom von Anderen festgestellt sind, zwingt zu dem Schlusse, dass additionelle Verbindungen bei der Umlagerung als Zwischenglieder nicht anzilnehmen sind, ansser man wollte jenen im Entstehungszustande ganz andere Eigenschaften zuschreiben als solche, die sie sonst besitzen.

Schliesst man diese Annahme ans, so bleibt keine andere ubrig, als die einer katalytischen Wirkung, welche die Processe, bei welchen die Maleînäure unter Aufnahme von Wasser, den Halogenwasserstoffsäuren, den Halogenen in gesättigte Verbindungen ibbergeht, auf die Maleïnsäure üben und sie zur Umlagerung, d. i. zum Übergang in die Fumarsäure zwingen. Unter diesem einheitlichen Gesichtspunkt lassen sich wohl die meisten der bisher bekannt gewesenen Übergangsreactionen zusammenfassen.

Nachdem weiter die Maleïnsäure nicht bloss durch Reactionen, bei denen sie theilweise andere Elemente, wie die des Wassers der Salzsäure etc. aufnimmat, sondern aich bei der Zerlegung ihrer Metallsalze durch Schwefelsäure und besonders durch Schwefelwasserstoff dieselbe Umlagerung in Fumarsäure erfährt und es sich gezeigt hat, dass bei allen diesen Reactionen definirbare Zwischenproducte wiederum ausgeschlossen bleiben, muss auch hier eî̀ katalytischer Einfluss rugestanden werden. Dasselbe gilt auch für die Wirkung, welche die chemischen Processe üben, die beim Zusammentreten von Schwefeldioxyd und Schwefelwasserstoff ablaufen.

Wabrscheinlich wird die relative Menge von Fumarsäure, die gebildet wird, mit dem Mass des Umsatzes chemischer Energie in einem gewissen Verbältnisse stehen, vorläufig fehlen jedoch die Daten, um diese Vermuthung auf ibre Richtigkeit zu prïfen.

Warum chemische Processe, wie die erwähnten, eine katalytische Wirkung üben, anderen, wie der Aufnahme von Wasser durch anhydridartige Körper eine solche abgeht, ist kaum zu entscheiden.

Ist das hier Vorgeführte richtig, so gilt es zweifellos nicht für den speciellen Fall der Umlagerung von Maleünsäure in Fumarsäure allein, sondern für eine grosse Zahl anderer Reactionen, 
vor Allem für die Umlagerungen der Homologen in der Reihe der Maleïn- und Fumarsänre, vielleicht für manche der ungesättigten Säuren, deren leichte Verwandlung in andere neuestens durch v. Baeyer und Fittig festgestellt wurde, vielleicht auch für so manche andere Reaction, die als stereochemischen Gründen wichtig: geworden ist, wie die Umlagerung von Oximen und deren Estern.

Es wäre darum von grosser Wichtigkeit, wenn dem Auftreten parallel lanfender Reactionen und der Stabilität eventueller additioneiler Zwischenstufen bei anderer Gelegenheit Aufmerksamkeit geschenkt würde.

Wie mir vorkommt, lässt sich eines der bekanntesten Beispiele von Umlagerung, die des gelben Phosphors in rothen, durch verschiedene chemische Processe, wie bei der Darstellung: von Alkyljodiden, bei der Einwirkung von Halogenen, weiter die Abscheidung von amorphem Schwefel aus Sulfiden gelegentlich deren Oxydation, am besten erklären, wenn man auch bei diesen annimmt, dass z. B. die chemische Umsetzung zwischen Phosphor, Jod und Alkobol die katalytische Ursache des Überganges in die allotrope Modification ist.

Auf dem Boden stereochemischer Anschanungen kaun doch ein principieller Unterschied zwischen dem Übergang von Maleïnsäure in Fumarsäure und der Verwandlung eines Elementes in die allotrope Modification kaum gezogen werden.

Vielleicht ist anch die Vermuthung richtig, dass es auf den katalytischen Einfluss chemischer Processe zurückzufïhren ist, wenn bei Substitutionsprocessen je nach Umständen verschiedene Isomere entstehen.

Die bisher vorgeführten Thatsachen sind nicht im Stande zu entscheiden, ob die Verschiedenheit zwischen der Maleïn- und der Fumarsäure structurchemisch oder stereochemisch ihren Grund hat, d. h. ob die le Bel-van t'Hoff'sehen Formeln oder die von Anschittz verfochtenen Geltung haben, und der sfrenge Beweis wird uberhaupt nicht leicht $\mathrm{zu}$ erbringen sein.

Auf dem Boden der Anschiitz'schen Vorstellungen kann man mit Consequenz einen katalytischen Einfluss ablehnen und sich mit additionellen Zwischenverbindungen behelfen, die von vornherein sebr unbeständig sind, deren Abwesenheit zu constatiren also belanglos ist. 
Gegen die Laktonformel ron Anschïtz spricht nun einmal die Thatsache, dass Maleïnsäure, wie ich fand, bei nicht allzu langem Erwärmen mit wässerigem oder alkoholischem Ätzkali so gut wie nicht verändert wird, also unter diesen Umständen die Laktonverbindung, die nach der Annahme von Anschütz durch Säuren leicht gelöst wird, intact bleibt, das anderemal das Verhalter der Ester der Maleïnsäure. Anschütz selber hat vor längerer Zeit gefunden, dass Maleïnsäuremethylester, mit Jod erhitzt, in Fumarsäureester übergeht.

Dasselbe habe ich für den $p$-Brombenzylester constatiren können, dessen Krystallisationsvermögen den Nachweis sehr erleichtert. Der bei $80-81^{\circ}$ schmelzende Maleïnsäureester geht in den bei $114-115^{\circ}$ schmelzenden Fumarsäureester nicht über, wenn er in festem Zustand oder mit Alkohol oder Eisessig bis 10 Stunden auf $150-160^{\circ}$ erhitzt wird, wohl aber, wenn den Fliissigkeiten etwas Jod zugesetzt ist.

Dieselbe Umwandlung geht schon in der Kälte und sehr rasch (im Verlauf weniger Stunden) vor sicb, wenn man den Ester mit einer Lösung von Chlorwasserstoffgas in einem Gemisch von Eisessig mit etwas Essigsäureanbydrid übergiesst. Diese Lösung verwandelt auch Maleïnsäure in Fumarsäure, was dafür spricht, dass Wasser zur Umwandlung nicht nöthig ist.

Die Verwandlung des Esters erfolgt in der Kälte dureh ein Gemisch von Eisessig, Essigsäureanhydrid und Salpetersäure nicht, ebensowenig wie die der Maleïnsäure, wohl aber beim Erhitzen auf $100^{\circ}$, und ersetzt man die Salpetersäure durch Schwefelsäure, so ist selbst bei $100^{\circ}$ eine Veränderung nicht zu beobachten.

Die Verhältnisse liegen beim Ester geradeso wie bei der freien Säure, und diese zeigt bei Abschluss von Wasser den genannten drei Mineralsäliren gegenüber dasselbe Verhalten wie in wässeriger Lösung. Demnach müssen alle die Erscheinungen einheitlich erklärbar sein.

Man kann nun allerdings auf Grund der von Ansehütz aufgestellten Theorien eine Erklärung finden, wie freie Malë̈nsäure bei An- oder Abwesenheit von Wasser durch Sänren in Fumarsänle übergeführt wird, man kann den ganz analogen Übergang ihrer Ester aber nicht oder nur mit Zuhilfenahme 
gewaltsamer Umlagerungen begreiflich machen, bei denen katalytische Einflïsse kaum zu umgehen sind. Die Nothwendigkeit, solche anzunehmen, ist für sich natürlich noch kein Grund, die sterische Formel der Maleïnsäure ansser Zwcifel zu nehmen, sie benimmt aber in Verbindung mit anderen Bedenken der Laktonformel den hauptsächlichsten Vorzug, die Erscheinungen ausschliesslich structurchemisch erklären zu können.

Der Umstand, dass der Maleïnsäure- $p$-Brombenzylester durch Erhitzen nicht in den Fumarsäureester iiberzufuhren ist, veranlasste das Verhalten verschiedener Salze der Maleïnsäure bei erhöhter Temperatur zu prüfen.

Es geschah dies derart, dass Proben der früher auf ihr Verhalten gegen Schwefelwasserstoff untersuchten Salze erhitzt und in Röhrehen mit verdünnter Salpetersäure ỉbergossen wurden. Nach 3 stündigem Trocknen auf $130^{\circ}$ Iösten sich mit Ausnahme des Silbersalzes alle klar auf, nach weiterem 3stiindigen Trocknen auf $155^{\circ}$ schieden sie etwas Fumarsäure ab, doch in geringer Menge.

So zeigten:

$1 / 10 \mathrm{NaOH}$ verbrancht

$0 \cdot 2427 g$ Kupfersalz eine Umwandlung von $8 \cdot 6 \%$ $\left(2 \cdot 35 \mathrm{~cm}^{3}\right)$ $1 \cdot 1652 \mathrm{~g}$ Bleisalz $\quad " \quad$ " $5 \cdot 1 \%$ $\left(3 \cdot 55 \mathrm{~cm}^{3}\right)$ $0 \cdot 3703 g$ Zinksalz $" \quad " \quad 2 \cdot 0 \%$ $\left(0 \cdot 75 \mathrm{~cm}^{3}\right)$

Die Umwandlung ist viel geringer, als sie unter denselben Umständen bei freier Maleïnsäure eintreten würde.

Jene Salze, die beim Trocknen durch tiefergehende Zersetzung stärker zersetzt waren, enthielten auch mehr Fumarsäure, eine Thatsache, die sich den früher erwähnten enge anschliesst und weiter daftir spricht, dass die Umlagerung auf den katalytischen Einfluss chemischer Reactionen zurïckzufübren ist. 